\title{
An Automatic Process for Sample Return Missions Based on Dynamic Programming Optimization
}

\author{
A. Bellome ${ }^{1}$ and Joan-Pau Sanchez Cuartielles ${ }^{2}$ \\ Cranfield University, Cranfield, Bedfordshire, MK430AL, United Kingdom \\ Jose Ignacio Rico Alvarez ${ }^{3}$ \\ Cranfield University, Cranfield, Bedfordshire, MK430AL, United Kingdom \\ Hadrien AFSA ${ }^{4}$ \\ Cranfield University, Cranfield, Bedfordshire, MK430AL, United Kingdom \\ Stephen Kemble 5 \\ Cranfield University, Cranfield, Bedfordshire, MK430AL, United Kingdom \\ Leonard Felicetti ${ }^{6}$ \\ Cranfield University, Cranfield, Bedfordshire, MK430AL, United Kingdom
}

\begin{abstract}
This work describes a methodology to design sample return missions and rendezvous trajectories options towards cometary objects. These are visited through a succession of flybys with Solar System planets, on an overall Multiple Gravity Assist (MGA) transfer. The method is based upon dynamic programming in conjunction to a specific MGA trajectory optimization model to investigate sample return mission scenarios. The model implemented is based on evaluation of grids of transfers between successive planets. The grid is obtained with Lambert arc transfer for a range of departure dates at one planet and range of time of flight to the next planet. For each successive planet in the sequence, discontinuities between incoming and outgoing Lambert arcs arise, which are in part compensated by the fly-by of the planet and, if required, an additional $\Delta \mathrm{v}$ maneuver is added on the given leg of a planet-toplanet transfer. The solutions identified are validated by re-optimizing the complete MGA trajectories as sequences of swing-bys, Deep Space Maneuvers and Lambert arcs transfers. A procedure for discontinuities removal using position constraints is also presented. Mission
\end{abstract}

\footnotetext{
${ }^{1}$ Ph.D. Candidate in Aerospace Engineering, Space Research Group, Centre for Autonomous and Cyber-Physical Systems, Cranfield University, United Kingdom.

${ }^{2}$ Lecturer in Space Engineering, Space Research Group, Centre for Autonomous and Cyber-Physical Systems, Cranfield University, United Kingdom.

${ }^{3}$ M.Sc. Student in Astronautics and Space Engineering, Space Research Group, Centre for Autonomous and CyberPhysical Systems, Cranfield University, United Kingdom.

${ }^{4}$ M.Sc. Student in Astronautics and Space Engineering, Space Research Group, Centre for Autonomous and CyberPhysical Systems, Cranfield University, United Kingdom.

${ }^{5}$ Visiting Research Fellow, Space Research Group, Centre for Autonomous and Cyber-Physical Systems, Cranfield University, United Kingdom.

${ }^{6}$ Lecturer in Space Engineering, Space Research Group, Centre for Autonomous and Cyber-Physical Systems, Cranfield University, United Kingdom.
} 
scenarios towards Saturn are used to validate the accuracy of proposed methods. Trajectory design for novel sample return options and rendezvous are explored for objects among Jupiter Family Comets (JFCs), as well as for never explored targets and orbital regions, as highly inclined Centaurs objects.

\section{Introduction}

Comets represent the trace of primitive Solar System formation processes. These objects are usually icy bodies that outgas when passing close to the Sun. Apart from water ice, cometary nuclei contain a variety of organic compounds, which may include methanol, ethanol, ethane and possibly long-chain hydrocarbons and amino acids. The comets exploration begun in the 80's with the close passage of Halley comet performed by Giotto mission [1], which showed for the first time the shape of a comet nucleus and found the first evidence of organic material in a comet. NASA's missions Deep Space 1 [2], Stardust [3] and Deep Impact [4] studied the geology, bulk properties of cometary nuclei, the nature of cometary dust and the cometary activity for comets 19P/Borrelly, 81P/Wild and 9P/Tempel 1 and 103P/Hartley, respectively. Stardust was the first mission that returned to Earth with dust samples collected from the coma of a cometary object, namely 81P/Wild. ESA's Rosetta mission [5] performed a rendezvous with comet 67P/ Churyumov-Gerasimenko, with the aim of studying the origin of comets, the relationship between cometary and interstellar material and its implications with regard to the origin of the Solar System.

Despite the success of past cometary missions, important questions in cometary science and Solar System formation remain open. These mainly regard the assembly processes of cometary materials and their relation with large-scale mixing in the antient Solar System, the role of comets in delivering volatiles and prebiotic compounds to early Earth, as well as the interaction between dust coma, surrounding plasma and nucleus [6]. Many of these questions do require cometary sample analysis with techniques only available on Earth, leading the scientific community to consider missions to return stored sample of cometary nucleus to Earth as a cornerstone in the extra-terrestrial exploration. Moreover, missions that can perform the rendezvous with new classes of comets, as main belt comets and Centaurs, can further enhance the understanding of Solar System formation and history, as well as providing a neverreached point of view of the out-of-the-ecliptic space, as in the case of highly inclined Centaur objects.

An obvious choice for designing rendezvous and sample return missions to those classes of comets relies on Multiple Gravity Assist (MGA) trajectories. In interplanetary missions, MGA transfers make use of successive passages, also called swing-bys or fly-bys, with planets to change the spacecraft heliocentric velocity. This permits to gain or lose energy with no propellant expenditure, thus allowing to explore cometary regions that would be demanding to reach otherwise. Although extensive literature exists addressing the problem of designing MGA transfers [7-9], the number of fly-bys that are required to perform a sample return mission are generally higher than any interplanetary transfers towards Solar System planets considered. This implies a huge number of design parameters, such as the presence of Deep Space Maneuvers (DSMs), fly-by parameters, encounter dates etc. In addition, current approaches often struggle to obtain true Pareto sets that reflect the multi-objective nature of the problem.

The present work proposes a novel approach based on multi-objective dynamic programming to automatically construct long MGA sequences for sample return missions towards cometary objects. The aim is to robustly solve the automatic MGA trajectory design, i.e., finding feasible sequences of planetary encounters and Pareto fronts of trajectories for the given sequence. Robustness is here assured by means of a quasi-systematic search of feasible MGA transfers, in addition to a specific MGA trajectory optimization model to investigate sample return mission scenarios. The MGA sequencing problem is here solved with Tisserand graph exploration, while the optimization model uses approximated $\Delta v$ occurring right at each swing-by planet, referred as infinity velocity defect. This allows for significant reduction of optimization parameters, while maintaining good representation of the actual design space. This also allows to obtain wide Pareto sets for missions of interest, approximating any maneuver required during the mission.

The paper articulates as follows: II describes the approach followed to automatically construct MGA transfers; section III describes the dynamic programming approach used to construct sample return missions; section IV focuses on the search for possible MGA transfer options for missions towards Saturn, used as a validation case, and cometary objects.

\section{Multiple Gravity Assist Trajectory Design}

The MGA trajectory design is a global optimization problem in its nature, as for a given trajectory option, namely a planetary sequence, there exist several locally optimal trajectories, in terms of planet phasing, presence of DSMs, 
etc. Designing an MGA transfer corresponds to solve a Mixed-Integer Non-Linear Programming (MINLP) problem [10], as it involves the optimization of both integers and continuous variables. The first correspond to the unknown planetary sequence, the latter to specific events like the departing date, transfer times, fly-by parameters and so on. The combination of discrete and continuous variables forms a challenging MINLP problem, as a variation of even a single planet implies a considerably different optimal transfer. For the present paper, Tisserand graphs are used to assess the feasibility of solving the first challenge, i.e., finding suitable planetary sequence to reach desired regions of the Solar System. A method based on successive evaluations of Lambert arcs linked at planetary encounters is then used to find trajectories between identified planets.

\section{A. Tisserand Graphs Exploration}

Designing an optimal MGA trajectory constitutes a MINLP problem that can be automatized by complex algorithms that go through combinations of planets, departure dates, time of flights, etc. in an exponentially increasing search space. To simplify the problem and guide the search, Tisserand graphs are used to perform a preliminary analysis of multi-gravity assist trajectories, within a circular and coplanar 2-body model.

A Tisserand graph is a visual tool [11] which can be used in interplanetary mission design for quick assessment of feasibility of different gravity-assist sequences. It has been used over the past decades for trajectory analysis and design of interplanetary missions. Some examples include the Galileo Orbiter's trajectory design [12], as well as Europa Orbiter design $[13,14]$. Tisserand graph has also been employed for building the winning trajectory of the $6^{\text {th }}$ edition of the Global Trajectory Optimization Competition (GTOC) [15]. Several modified versions were developed to adapt it to specific purposes, as the Saturn moon tours [16], the case of circular-restricted 3-body problem [17], and low-thrust propulsion trajectories [18].

Tisserand graphs can be obtained by parametrizing the Tisserand invariant with respect to the spacecraft relative velocity to the gravity assist body, also called infinity velocity $\vec{v}_{\infty}$. It is thus possible to visualize how a gravity assist changes the orbit of the spacecraft relating the Tisserand invariant and the turning angle $\alpha$ (see Fig. 1) with the resulting orbit of the spacecraft.
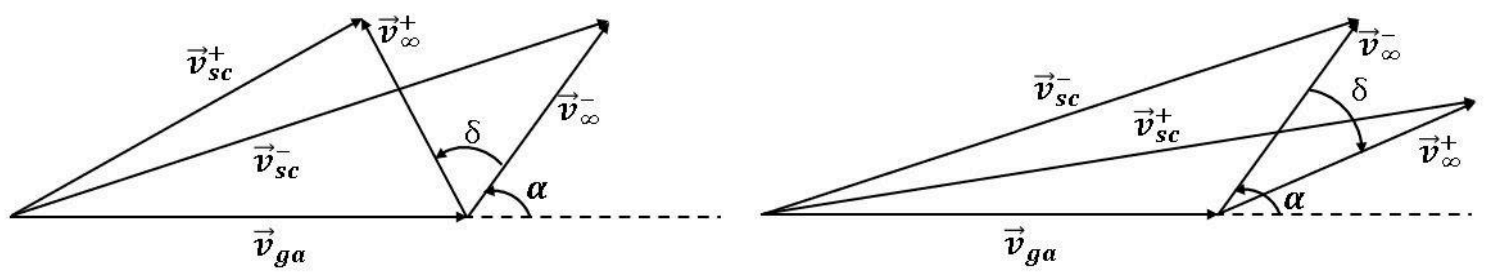

Fig. 1 Vector diagram representing the effect of the fly-by in front (left image) and behind (right image) a given gravity assist body. Left and right cases result in minimizing and maximizing the energy after the swing-by, respectively.

The vector diagram in Fig. 1 represents the effect of a close passage with a generic planet with velocity $\vec{v}_{g a}$. The spacecraft velocities before and after the fly-by are $\vec{v}_{s c}^{-}$and $\vec{v}_{s c}^{+}$, respectively. The turning angle $\delta$ determines the orbit of the spacecraft after the flyby, rotating the infinity velocity vector from $\vec{v}_{\infty}^{-}$to $\vec{v}_{\infty}^{+}$. The flyby is assumed to occur instantaneously when compared to the interplanetary travel times. 


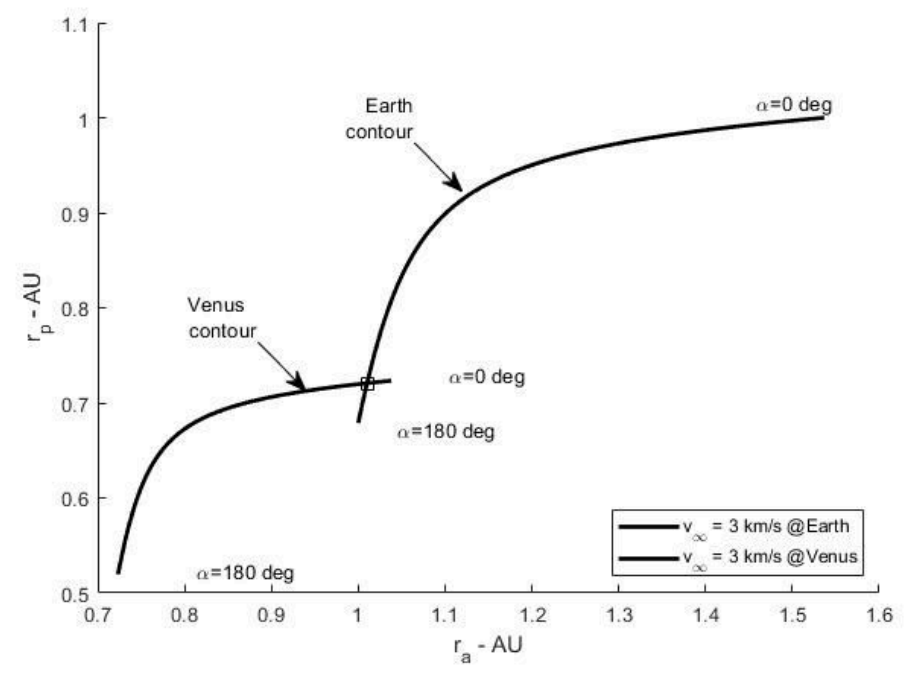

Fig. 2 Infinity velocity contours of $3 \mathrm{~km} / \mathrm{s}$ at Venus and Earth, respectively. Tick marks separate flybys with $200 \mathrm{~km}$ altitude. The squared mark represents a possible transfer between the two planets.

For different values of $\alpha$ and $\vec{v}_{\infty}$ magnitudes, one obtains different orbits, that can be represented in a map as in Fig. 2, representing the spacecraft orbits as function of $r_{a}$ and $r_{p}$, i.e., apoapsis and periapsis, respectively. If $\alpha=$ $0 \mathrm{deg}$, then the spacecraft velocity is aligned with the planet one, corresponding to the highest orbital energy for a given $\vec{v}_{\infty}$ magnitude (see also Fig. 2); if $\alpha=180 \mathrm{deg}$, the spacecraft velocity is antiparallel to the planet's one, resulting in the lowest orbital energy for the given $\vec{v}_{\infty}$ magnitude. Intersections between different contours represent possible transfer orbits between two planets. However, this opportunity only exists from an energy point of view, since Tisserand graphs contain no explicit information regarding the planetary phase and transfer time. Thus, the exploration of a Tisserand graph consists in evaluating the effect of all the possible sequences of planetary swing-bys in the parameters of the Tisserand invariant, i.e., $r_{a}$ and $r_{p}$. It is thus possible to automatically enumerate all the planetary sequences and excess velocities which are energetically feasible to reach the desired target orbit by connecting different contours when intersections occur. A Depth First (DF) [19] algorithm is used for this purpose. A node in the search space is defined as a given position in the Tisserand map (i.e., $r_{a}$ and $r_{p}$ ), corresponding to a heliocentric orbit. Once a starting node is defined, the second level (i.e., the set of potential reachable new nodes) is constructed by evaluating all the possible planetary swing-bys from the given departing condition. This is done by considering the intersections between the current node and the orbits of all the planets in the Solar System. If the current node defines an orbit that crosses the orbit of a planet, then a swing-by with this given planet is possible. Successive levels are built by checking all the possible flybys from each resulting orbit from the previous level. The search is stopped either when the arrival node or a maximum number of levels (i.e., iterations) is reached.

Table 1. Common resonances for innermost Solar System planets

\begin{tabular}{|c|c|}
\hline Planet & Resonances available \\
\hline Mercury & $1: 1,6: 5,5: 4,4: 3,3: 2$ \\
\hline Venus & $1: 1,2: 1,3: 4,2: 3,1: 2$ \\
\hline Earth & $1: 1,2: 1,3: 1,3: 2,2: 3$ \\
\hline Mars & $1: 1,2: 1,3: 1$ \\
\hline
\end{tabular}

However, as the change of the orbit induced by a flyby is limited by the minimum altitude the spacecraft can have during the maneuver, a single flyby may be insufficient to reach the next planet. On the Tisserand graph, this implies that the orbit cannot shift enough along a contour to reach the next intersection. Therefore, one needs to prune paths with unfeasible legs or try to find reachable intermediate orbits on the contour, as with resonant transfers. These allow the spacecraft to perform a 360 degrees revolution around the Sun on a same planet-to-planet transfer. On resonant transfers, a ratio of integers exists between the planet and the spacecraft orbit periods. The ratio is expressed as $M: N$, where $M$ and $N$ are the number of planet and spacecraft revolutions, respectively. For Solar System planets, the most used resonances are listed in Table 1. As a result, a list of feasible fly-by sequences is conveniently obtained, as well as approximate infinity velocities at planetary encounters, useful to inform successive stage of trajectory optimization. 


\section{B. MGA Trajectory Optimization via Grid-based Multi Objective Tree Search}

Once planetary sequences and encounter velocities have been obtained, a method is used to find optimal trajectories. It employs evaluation of grids of transfers between successive planet fly-bys in an overall mission design. The grid is obtained by solving Lamberts problem from a range of start dates at one planet and a range of transfer durations to the next planet, for each successive pair of planets in the sequence.

The cost of an MGA planet-to-planet leg (without considering DSMs) could be either approximated by a powered fly-by or by a small maneuver applied after the first planet of the leg. A powered fly-by model assumes a $\Delta v$ manoeuvre at the pericentre of the incoming hyperbola to match the incoming and outgoing spacecraft velocities. However, this methos has not been implemented in the context of real interplanetary missions, due to navigation challenges that it arises. Therefore, $\Delta v$ are computed as defects between incoming and outgoing spacecraft relative velocities with respect to the planet, which are solutions of Lambert's problem for the given leg. These velocity discontinuities between legs are thus considered as impulsive maneuvers applied right after the planetary encounter.

The defects are thus computed as:

$$
\Delta v= \begin{cases}|| \vec{v}_{\infty}^{+}|-|\left|\vec{v}_{\infty}^{-}\right| & \text {if } \delta \leq \delta_{\max } \\ \sqrt{\left|\vec{v}_{\infty}^{+}\right|^{2}+\left|\vec{v}_{\infty}^{-}\right|^{2}-2\left|\vec{v}_{\infty}^{+}\right|\left|\vec{v}_{\infty}^{-}\right| \cos \left(\delta_{\max }-\delta\right)} & \text { otherwise }\end{cases}
$$

Where: $\vec{v}_{\infty}^{-}$and $\vec{v}_{\infty}^{+}$are the infinity velocities before and after the planetary encounter (see again Fig. 1) as coming from Lambert solutions; $\delta$ defines the change of direction between incoming and outgoing legs of the fly-by, such that as $\cos (\delta)=\frac{\vec{v}_{\infty}^{-} \cdot \vec{v}_{\infty}^{+}}{\left|\vec{v}_{\infty}^{-}\right|\left|\vec{v}_{\infty}^{+}\right|}$, with a positive 180-degree range. The maximum deflection is limited by the periapsis of the swing-by hyperbola $r_{p, \min }$, through $\delta_{\text {max }}=2 \operatorname{asin}\left(\left[1+\frac{r_{p, \min }\left|\vec{v}_{\infty}\right|^{2}}{\mu_{p l}}\right]^{-1}\right)$, where $\mu_{p l}$ is the gravitational parameter of the fly-by planet. Note that since $\vec{v}_{\infty}^{-}$and $\vec{v}_{\infty}^{+}$represent spacecraft velocities relative to the fly-by body, the $\Delta v$ as from eq. (1) ultimately depends upon planet ephemerides, through its heliocentric velocity at the encounter epoch. This allows for a great reduction of the design parameters, while maintaining a good representation for the search space, as any DSM is included in the search if sufficiently large defect is considered. The objective functions to be optimized are the total transfer time required for the MGA transfer and the total $\Delta v$ cost expressed as:

$$
f=\left|\vec{v}_{\infty, \text { dep }}\right|+\sum_{i=1}^{n} \Delta v_{i}+\left|\vec{v}_{\infty, a r r}\right|
$$

Where: $\vec{v}_{\infty, \text { dep }}$ and $\vec{v}_{\infty, \text { arr }}$ are the spacecraft velocities relative to departing and arrival object, respectively, and $\Delta v_{i}$ are the defects computed at each fly-by and $n$ is the total number of fly-bys. As the optimization is iterated along the MGA planets, the number of identified routes grows. If particularly long fly-by sequences are sought, the number of possible routes may potentially grow to unmanageable numbers. However, this problem is mitigated via the application of both a multi-objective Beam Search (MO-BS) [20] strategy and an incremental pruning to identify and filter out infeasible routes. Both these filters are based on heuristic information that can be directly related with practical mission design constraints, as the magnitude of the defects and overall mission duration.

On MO-BS, each node represents a transfer that can be incrementally constructed expanding one or more of its branches (i.e., adding a trajectory leg in an overall MGA sequence). In BS algorithms, the computational effort is bounded by employing heuristics that prevent the exploration of non-promising branches. In the present paper, the exploration of the trajectory options is performed one depth-level at a time. To preserve the multi-objective nature of the problem at hand, the pruning scheme introduced by the BS is applied together with Pareto dominance concepts, where the top nodes to be kept for further expansion are determined through non-dominated sorting. In MO-BS, the size of the beam, designated as the beam width, governs the selection process, as it represents the number of options retained for successive expansion. A full grid search, on which the beam width is the maximum allowed, carries forward all the solutions. This ultimately has computer memory issue. For the purposes of the present paper, a wide beam can carry forward more solutions with both larger defects and longer time of flights, for consideration in the final Pareto set after arrival at the target. On the other hand, a too narrow width will prune out local optimal solutions. Regarding constraints over the $\Delta v$ defects at each swing-by, these are linked to limitations on spacecraft propulsive system, thus large values are not feasible for standard spacecraft designs. To achieve computational efficiency, $\Delta v$ defects should be the minimum possible, but subject to the condition that locally optimal solutions are not lose and Pareto sets characteristics are retained. This implies an upper limit on the DSM in-between two consecutive swing- 
bys, which is related by the leveraging ratio, i.e., ratio between $\Delta v$ defect and precedent DSM (see also later section III and D). The leveraging ratio varies between typically 0.5 and 6.5 for the range of missions considered in the present paper; so, for example a maximum defect of $2000 \mathrm{~m} / \mathrm{s}$ implies a maximum DSM magnitude of about $310 \mathrm{~m} / \mathrm{s}$ in the extreme leveraging cases (note that 6.5 is still a high leveraging ratio and will be generally less than this). Therefore, in this paper, only $\Delta v \leq 2 \mathrm{~km} / \mathrm{s}$ are retained for further considerations. The MO-BS approach provides non-dominant Pareto front of mission overall duration and objective value as from eq. (2). However, $\Delta v$ defects are manoeuvres applied immediately after departing from a fly-by, and so they do not represent real maneuvers in the context of a mission design. Therefore, as a post-processing stage, these $\Delta v$ are converted to DSMs between two successive planets in the sequence. This is done employing the so-called MGA-DSM model [21] with a Particle Swarm Optimization (PSO).

\section{Defects Removal by DSM Correction Using Position Constraint}

Insertion of a DSM to remove an infinity velocity defect at the next fly-by is here used to establish the relationship between the maneuver and the corresponding correction of the defect. The incoming defect dependency on a preceding mid-course DSM is obtained. We refer to this dependency as leveraging ratio, i.e., the ratio between the defect and a precedent DSM. A key consideration is the maintenance of the subsequent rendezvous with the target planet for the fly-by, as well as the removal of the defect. The DSM is assumed to take place at a time $t_{0}$ on a planet-to-planet leg, while the successive planetary encounter occurs at $t_{f}$. The DSM is derived in a reference frame which has $\hat{v}_{v}, \hat{v}_{p}$ and $\hat{v}_{n}$ as unit vectors, which are components along the velocity vector, in-orbit plane perpendicular to the velocity vector and out-of-plane normal, respectively. The DSM has an impact on the position vector achieved at the epoch of the flyby. Therefore, a constraint vector $\vec{C}$ should be considered that maintains the relative position error with respect to the swing-by planet and the infinity velocity defect at zero. The constraints are thus $\vec{C}=\left[\vec{r}_{r e l}, \Delta v_{\infty}\right]^{T}$, on which $\vec{r}_{\text {rel }}=$ $\vec{r}-\vec{r}_{P L}$, is the difference between the spacecraft and planet position vectors at $t_{f}$ (i.e. $\vec{r}$ and $\vec{r}_{P L}$, respectively), and $\Delta v_{\infty}$ is the infinity velocity defect at $t_{f}$. The control variables are thus $\vec{U}=\left[\Delta v\left(t_{0}\right), t_{f}\right]^{T}$, on which $\Delta v\left(t_{0}\right)=$ $\left[D S M_{v}, D S M_{p}, D S M_{n}\right]^{T}$ is the manoeuvre vector, written in the reference frame identified by $\hat{v}_{v}, \hat{v}_{p}$ and $\hat{v}_{n}$, as defined above.

Therefore, the control law is:

$$
\frac{\partial \vec{C}}{\partial \vec{U}} \Delta \vec{U}=\left[\begin{array}{c}
0 \\
0 \\
0 \\
-\Delta v_{\infty}
\end{array}\right]
$$

From which the control increment $\Delta \vec{U}$ is found via matrix inversion. From equation ( 3 ), the vector $\left[0,0,0,-\Delta v_{\infty}\right]^{T}$ corresponds to the required change in the constraint vector $\vec{C}$, and the matrix $\frac{\partial \vec{C}}{\partial \vec{U}}$ is defined as follows:

$$
\frac{\partial \vec{C}}{\partial \vec{U}}=\left[\begin{array}{cc}
\frac{\partial \vec{r}_{r e l}\left(t_{f}\right)}{\partial \Delta \vec{v}\left(t_{0}\right)} & \frac{\partial \vec{r}_{r e l}\left(t_{f}\right)}{\partial t_{f}} \\
\frac{\partial \vec{v}_{r e l} \mid\left(t_{f}\right)}{\partial \Delta \vec{v}\left(t_{0}\right)} & 0
\end{array}\right]
$$

Where $\vec{v}_{r e l}=\vec{v}-\vec{v}_{P L}$, is the spacecraft velocity vector relative to the fly-by planet computed at time $t_{f}$ ( $\vec{v}$ and $\vec{v}_{P L}$ are the spacecraft and planet velocities at $t_{f}$, respectively). By computing the State Transition Matrix (STM) $\Phi$ between $t_{0}$ and $t_{f}$, one has:

$$
\Phi=\left[\begin{array}{ll}
\frac{\partial \vec{r}\left(t_{f}\right)}{\partial \vec{r}\left(t_{0}\right)} & \frac{\partial \vec{r}\left(t_{f}\right)}{\partial \vec{v}\left(t_{0}\right)} \\
\frac{\partial \vec{v}\left(t_{f}\right)}{\partial \vec{r}\left(t_{0}\right)} & \frac{\partial \vec{v}\left(t_{f}\right)}{\partial \vec{v}\left(t_{0}\right)}
\end{array}\right]
$$

Using $\frac{\partial \vec{r}_{r e l}\left(t_{f}\right)}{\partial \vec{v}\left(t_{0}\right)}=\frac{\partial \vec{r}\left(t_{f}\right)}{\partial \vec{v}\left(t_{0}\right)}$, then: 


$$
\frac{\partial \vec{r}_{r e l}\left(t_{f}\right)}{\partial \Delta \vec{v}\left(t_{0}\right)}=\left[\frac{\partial \vec{r}\left(t_{f}\right)}{\partial \vec{v}\left(t_{0}\right)} \hat{v}_{v} \frac{\partial \vec{r}\left(t_{f}\right)}{\partial \vec{v}\left(t_{0}\right)} \hat{v}_{p} \frac{\partial \vec{r}\left(t_{f}\right)}{\partial \vec{v}\left(t_{0}\right)} \hat{v}_{n}\right]
$$

Moreover, one has:

$$
\frac{\partial \vec{r}_{r e l}\left(t_{f}\right)}{\partial t_{f}}=\vec{v}\left(t_{f}\right)-\vec{v}_{P L}\left(t_{f}\right)
$$

Where $\vec{v}$ and $\vec{v}_{P L}$ are again the spacecraft and planet velocities computed at $t_{f}$, respectively. Then, to evaluate the following:

$$
\frac{\partial\left|\vec{v}_{r e l}\right|\left(t_{f}\right)}{\partial \Delta \vec{v}\left(t_{0}\right)}=\left[\frac{\partial\left|\vec{v}_{r e l}\right|\left(t_{f}\right)}{D S M_{v}} \frac{\partial\left|\vec{v}_{r e l}\right|\left(t_{f}\right)}{D S M_{p}} \frac{\partial\left|\vec{v}_{r e l}\right|\left(t_{f}\right)}{D S M_{n}}\right]
$$

One uses:

$$
\frac{\partial\left|\vec{v}_{r e l}\right|\left(t_{f}\right)}{\partial D S M_{i}}=\frac{\partial\left(\vec{v}\left(t_{f}\right)-\vec{v}_{P L}\left(t_{f}\right)\right)}{\partial D S M_{i}} \cdot \hat{v}_{r e l}=\frac{\partial \vec{v}\left(t_{f}\right)}{\partial D S M_{i}} \cdot \hat{v}_{r e l}
$$

On which $\frac{\partial \vec{v}\left(t_{f}\right)}{\partial D S M_{i}}=\frac{\partial \vec{v}\left(t_{f}\right)}{\partial \vec{v}\left(t_{0}\right)} \cdot \hat{v}_{i}$, for each $i=v, p, n$. Therefore, it is possible to compute $\frac{\partial \vec{C}}{\partial \vec{U}}$ as defined in (2) using equations (4), (5) and ( 6), and thus $\Delta \vec{U}$ from ( 1). This is again evaluated via STM along the nominal trajectory for different values of $t_{0}$. The leveraging ratio $\Delta v_{\infty} /|\Delta \vec{v}|$ can also be derived as a function of $t_{0}$. Its maximum value, corresponding to the minimum $|\Delta \vec{v}|$ over the trajectory, can then be obtained. The terms $\frac{\partial \vec{r}\left(t_{f}\right)}{\partial \vec{v}\left(t_{0}\right)}$ and $\frac{\partial \vec{v}\left(t_{f}\right)}{\partial \vec{v}\left(t_{0}\right)}$ used in equations (4) and (6) are obtained from the standard STM as in equation ( 3 ) for the trajectory between $t_{0}$ and $t_{f}$.

\section{Application of Bellman's Principle of Optimality to Sample Return Missions}

To look for optimal trajectories in the context of sample return missions with MGA transfer, a dynamic programming approach has been developed. A dynamic programming algorithm benefits from a description of optimization problems such that Bellman's principle of optimality [22] is true or holds. Such principle states that an optimal policy has the property to be independent from initial state and decisions. This means that the optimal policy is the same even if the optimal control is found starting at intermediate states. Here, by optimal policy is meant a sequence of decisions which is the most advantageous from a preassigned criterion. Translated to the MGA trajectory design, Bellman's principle of optimality would state that, regardless of the object at which the spacecraft is, the optimal set containing this specific object would contain the optimal subset of objects before and after the visited one. Here, optimality is again referred to multi-objective criteria, considering both mission duration and propellant consumption. This principle thus allows to apply tree search procedure as described in section B at more local level.

In the context of comet sample return missions with rendezvous, on which the spacecraft velocity must match the one of the selected objects, given a set of comets of interest, the following approach can find among them those with feasible trajectories for a particular planet-comet-planet $\left(\mathrm{P}-\mathrm{C}-\mathrm{P}^{\prime}\right)$ sequence. Planets before and after the cometary encounter are detected with Tisserand map.

The problem can be formulated as:

Find $x \in I$

$$
\text { That minimizes } \Delta v(\boldsymbol{x})=\Delta v_{1}+\Delta v_{2}
$$

Where: $\boldsymbol{x}=\left[t_{0}, T_{1}, T_{\text {ops }}, T_{2}\right], t_{0}$ being the departure epoch at last planetary encounter before the cometary rendezvous, $T_{1}$ the duration needed to travel along the conic arc joining the planet and the comet, $T_{\text {ops }}$ the duration of the operations phase at cometary rendezvous, and $T_{2}$ the time needed to travel along the conic arc joining the comet at the end of the operations phase and the arrival planet; $I=I_{0} \times I_{1} \times I_{\text {ops }} \times I_{2}$ is an hyperrectangle in $\mathbb{R}^{4}$ representing the domain for $\boldsymbol{x} ; \Delta v_{1}$ is the impulse required to capture comet's orbit and $\Delta v_{2}$ is the impulse necessary to depart from 
comet's orbit at the end of the operations phase. The idea is to decompose the $\mathrm{P}-\mathrm{C}-\mathrm{P}$ ' in two bidimensional uncoupled subproblems, i.e., $\mathrm{P}-\mathrm{C}$ and $\mathrm{C}-\mathrm{P}$ ', linked by the operations phase in the vicinity of the comet's orbit. Consequently, $I=I_{0} \times I_{1} \times I_{\text {ops }} \times I_{2}$ can be transformed into $I^{\prime}=I_{0} \times I_{1} \cup I_{\text {ops }} \times I_{2}$ as from Fig. 3, where $t_{1}$, $t_{\text {ops }}$ and $t_{2}$ are now the epochs at which the comet is encountered, left and the next planet is flown-by, respectively.

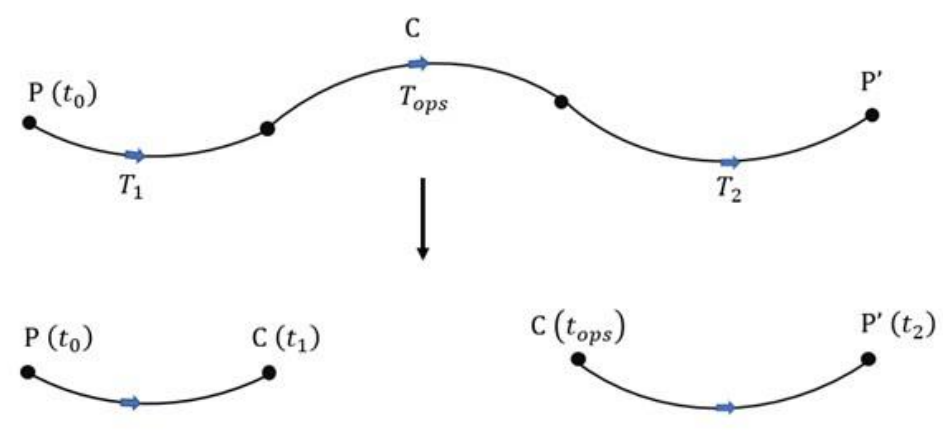

Fig. 3 Decomposition of the P-C-P' problem.

All the potential transfers in terms of $\mathrm{P}-\mathrm{C}$ and $\mathrm{C}-\mathrm{P}^{\prime}$ are computed over grid of departing and arrival epochs $t_{0}$ and $t_{1}$. This is done for all the combination of planets $\mathrm{P}$ and $\mathrm{P}$ ' identified by the Tisserand map and for all the comets $\mathrm{C}$ of interest. All the solutions obtained are then filtered to find among them all the transfers achieved by an impulse less than a given restriction $\Delta v_{\max }$. It must be noticed that the restriction corresponds to the maximum $\Delta v$ allowed for each subproblem, $\Delta v_{i}<\Delta v_{\max }$ where $i=1,2$. At this point, a given comet may be discarded if it is not accessible by any transfer that fulfils this requirement. Filtered solutions conform the search space assessed throughout the rest of the procedure. Then, all comets with feasible transfers for a particular $\mathrm{P}-\mathrm{C}$ and $\mathrm{C}-\mathrm{P}$ ' subproblem are considered. Among them, one finds those comets with viable transfers for both subproblems. Hence, these bodies are considered as potential candidates to accomplish the complete $\mathrm{P}-\mathrm{C}-\mathrm{P}^{\prime}$ sequence. The process is repeated until all the different sequences of interest are analyzed. It is now possible to obtain optimal trajectories for all the $\mathrm{P}-\mathrm{C}-\mathrm{P}^{\prime}$ sequences under study.

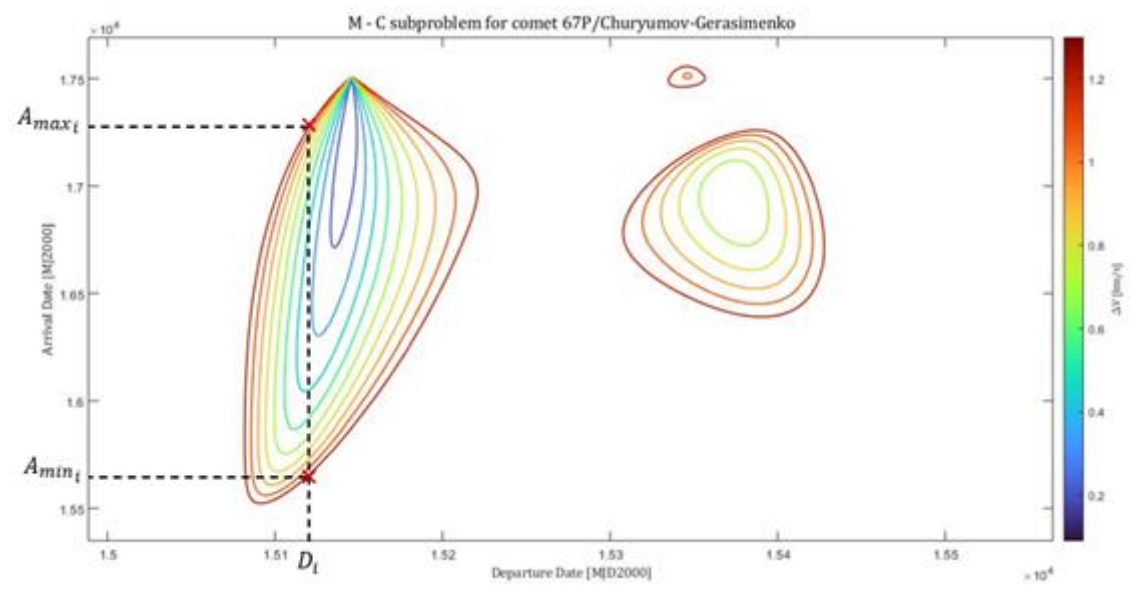

Fig. 4 Pork-chop plot for Mars - 67P leg in the 2030-2050 timeframe.

One first considers the $\mathrm{P}-\mathrm{C}$ subproblem. This can be represented in a pork-chop plot as in Fig. 4, on which an example for rendezvous comet 67P/Churyumov-Gerasimenko is shown. The pork-chop is with respect to the departure epoch until a feasible leaving date from departure planet $D_{i}$ is found (see Fig. 4). Minimum and maximum admissible arrival date to the comet, i.e., $A_{\min _{i}}$ and $A_{\max _{i}}$, respectively from Fig. 4. These two values comprise the region of allowed arrival dates to the comet, for a given $D_{i}$. Thus, any arrival date to the comet $A_{j}$, must obey: $A_{j} \in$ $\left[A_{\min _{i}}, A_{\max _{i}}\right]$. Now, the second subproblem is analysed, exemplified by its pork-chop plot in Fig. 5. 


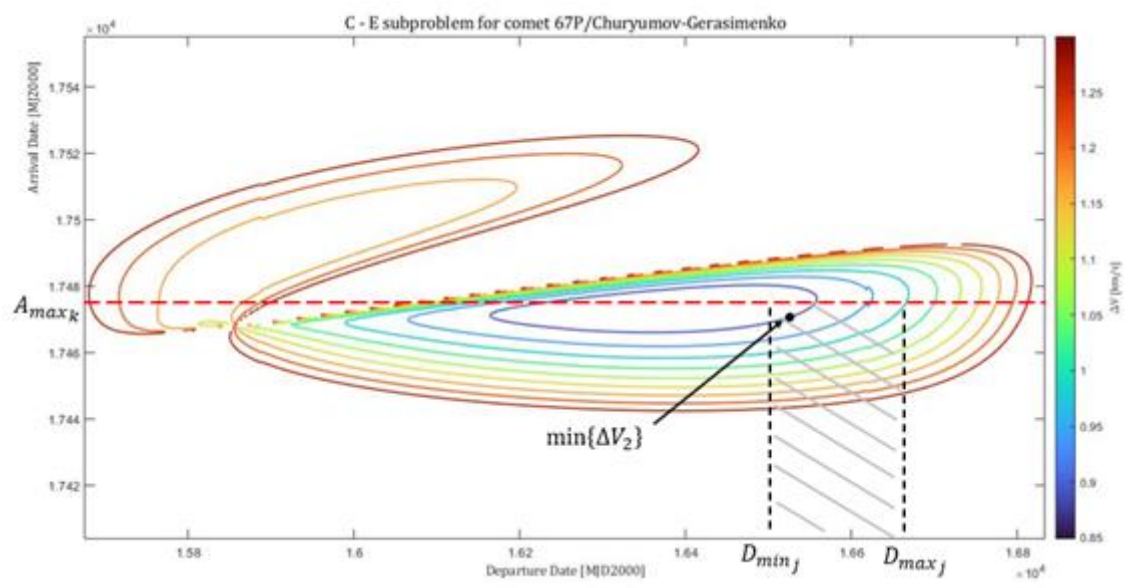

Fig. 5 Pork-chop plot for Earth - 67P leg in the 2030-2050 timeframe.

For each $A_{j} \in\left[A_{\text {min }_{i}}, A_{\text {max }_{i}}\right]$, the minimum and maximum allowed departure dates from the comet are defined, i.e. $D_{\min _{i}}$ and $D_{\max _{i}}$, respectively, from Fig. 5. These two values depend on the minimum and maximum allowed duration for the operations phase within comet's orbit. The stripped region from Fig. 5 is scanned to find the minimum $\Delta v_{2}$. This value dictates the departure date from the comet $D_{j}$ and the date when the arrival planet is intercepted $A_{k}$. The whole procedure is repeated for the next feasible $D_{i}$ until all the possible solutions are assessed.

Once the $\mathrm{P}-\mathrm{C}-\mathrm{P}$ ' problem is thus solved, methods described in section $\mathrm{A}, \mathrm{B}$ and $\mathrm{C}$ are used to construct full MGA trajectories for sample return mission. Since the $\mathrm{P}-\mathrm{C}$ and $\mathrm{C}-\mathrm{P}$ ' phases are now considered fixed, one expands the tree of possible fly-by options backward in time, i.e., from the last available planet $\mathrm{P}$ before the cometary encounter to Earth departure, and forward in time, i.e., from the first planet P' after the cometary departure to Earth arrival. Fig. 6 illustrates the application of Bellman's principle of optimality applied to the comet sample return problem. Once the intermediate $\mathrm{P}-\mathrm{C}-\mathrm{P}$ ' problem is defined, the (Pareto-)optimal sub-sequences can be obtained in backward and forward sides, and thus the overall sample return trajectory is obtained.

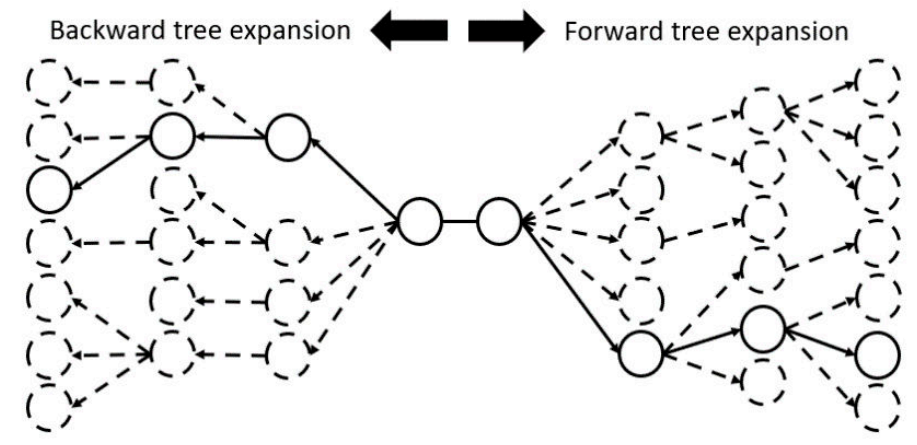

Fig. 6 Backward and forward tree expansion for a given comet sample return mission. Central left node corresponds to cometary rendezvous, while central right node to cometary departure on the return phase. Bold nodes and branches belong to optimal sequence.

\section{Results}

Optimal transfers are determined for benchmark missions as well as for building novel transfers for sample return options. An MGA mission towards Saturn is firstly tested referring to ESA's Cassini mission [23] towards Saturn, to verify the ability to reproduce past mission transfers, as well as to discuss the capability of the procedure as in sections $\mathrm{A}$ and $\mathrm{B}$ to approximate any maneuver required during the mission. Moreover, sample return mission options are presented for objects among the Jupiter Family Comets (JFCs) and Centaurs in the context of practical mission design. 


\section{Missions towards Saturn}

Transfers towards Saturn are searched in the 1997 launch window, which is the one of Cassini mission [23]. Cassini performed successive close passages at Venus-Venus-Earth-Jupiter-Saturn (resulting in an EVVEJS sequence, the first planet being the departing one), launching in October 1997 and approaching Saturn in July 2004. This is known to be one of the most difficult impulsive thrust missions to find optimal transfers $[21,24,25]$, as large DSMs are needed to achieve Saturn with reduced $\Delta v$, while maintaining reduced transfer times. The mission required at least one large DSM between the two Venus fly-bys to reach its destination. In this case, the $\Delta v$ computed with the method described in section B may lead to larger values. This is because the $\Delta v$ identified in the preferred method are maneuvers applied immediately after departure from a fly-by, thus not representing DSMs in a real mission design context. In this case, a simple post-process computation as described at the end of section B is needed.

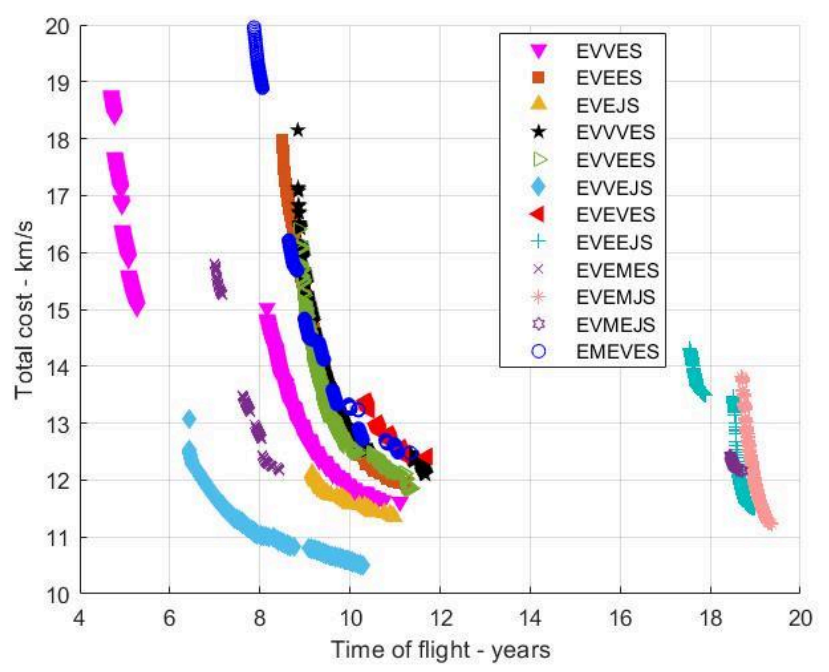

Fig. 7 Pareto fronts for sequences towards Saturn for departure date in 1997. Total cost refers to the sum of infinity velocities at departure and arrival planet and all the defects employed along the transfer.

This problem has been evaluated with a range of time constraints to consider the following scenarios:

- The actual Cassini mission, i.e., a fast transfer to Saturn of about 7 years.

- A test case reported in [26] with relaxed time constraints, i.e. solutions with about 9.9 years with a 2200 days upper limit on the last leg from Jupiter to Saturn, corresponding to the best-known time constrained transfer.

- A transfer without time constraints. Although this would not be a practicable mission design, it is of importance to understand the capability of the presented procedure to identify local and global optima.

In particular, Fig. 7 shows that Pareto front for sequence EVVEJS is comprehensive in that it correctly identifies both the fast transfer to Saturn, as actually employed for Cassini [23], and the best known solutions?

Details and trajectories representations can be found in Table 2 and Table 3, as well as in Fig. 8 and Fig. 9. Results indicate that the proposed procedure correctly identifies best-known solution, as launch and epochs of fly-bys correspond closely to within a day.

A key feature of the method described here is the relationship between the infinity velocity defects identified with the grid optimization and the DSMs identified in the refinement stage. Real world missions utilize DSMs, and these are inserted via the optimization procedure on the refinement step. The analytical approximation employed is described in section C. This allows the leveraging obtainable from a DSM to be evaluated, i.e., the dependency of an infinity velocity defect on a preceding DSM. As it can be seen from both Table 2 and Table 3, large defects occurring in the Venus-Venus leg of EVVEJS (both for actual Cassini and best-known solutions) are replaced by DSMs, reducing from about $1.9 \mathrm{~km} / \mathrm{s}$ to approximately $400 \mathrm{~m} / \mathrm{s}$. The $\Delta v$ defects occurring in all the other legs are reduced to zero.

\footnotetext{
${ }^{7}$ https://www.esa.int/gsp/ACT/projects/gtop/ , last accessed March 10, 2021
} 

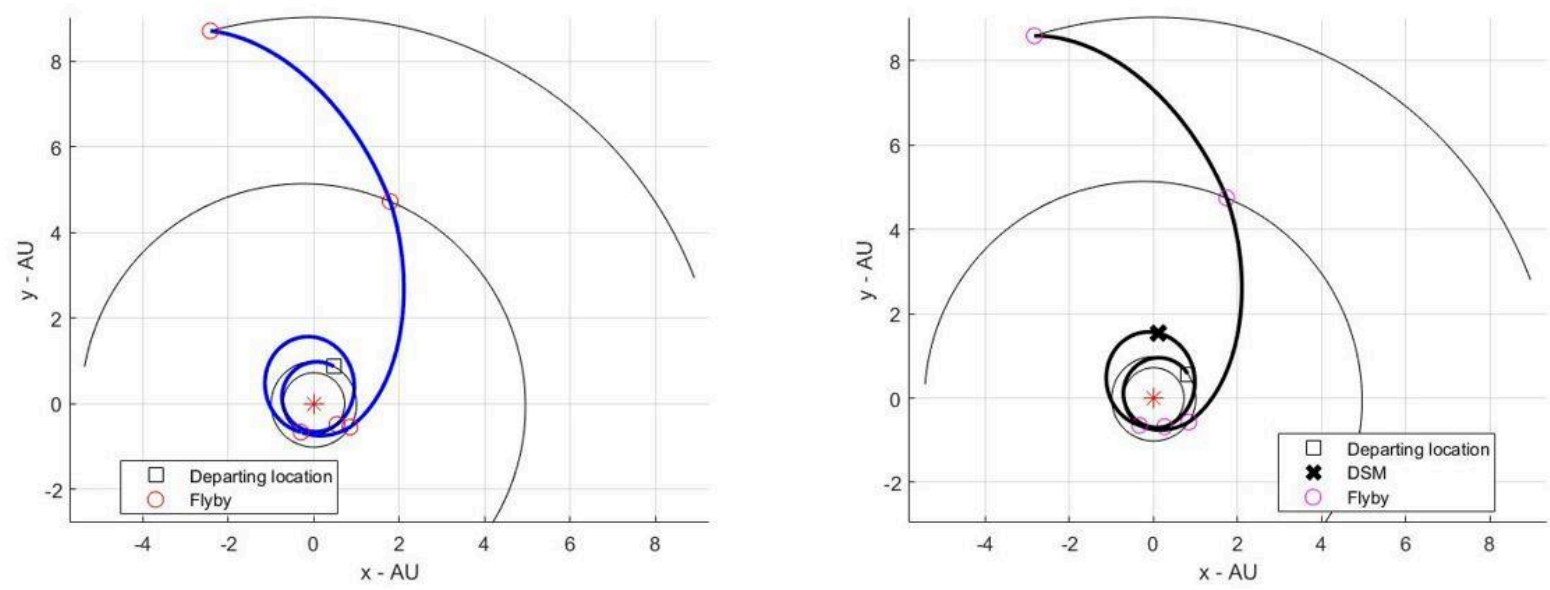

Fig. 8 Cassini-like EVVEJS transfer with departure date in 1997. Left plot is obtained with defects formulation, while right plot with DSMs formulation. From innermost to outermost, black circles represent Venus, Earth, Jupiter, and Saturn orbits, respectively.
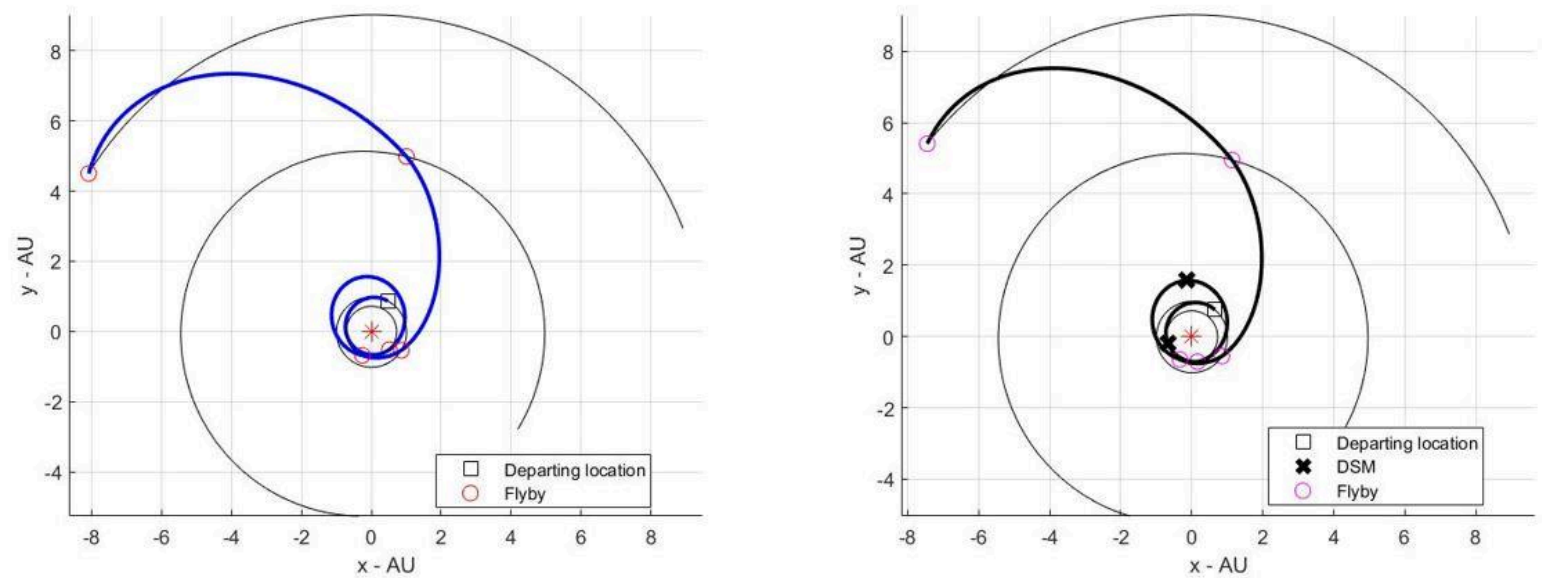

Fig. 9 Best-known solution for EVVEJS transfer with departure date in 1997. Left plot is obtained with defects formulation, while right plot with DSMs formulation. From innermost to outermost, black circles represent Venus, Earth, Jupiter, and Saturn orbits, respectively.

Table 2. Results for actual Cassini trajectory compared to grid optimization solutions in 1997 launch window. If a $\Delta v$ is not present on a given leg, a '--' is included in the table.

\begin{tabular}{|c|c|c|c|}
\hline Event & Actual Cassini & Grid Optimization & Refinement \\
\hline Launch & 6 October 1997 & 23 November 1997 & 20 October 1997 \\
\hline$C_{3}$ (departure) & $18.1 \mathrm{~km}^{2} / \mathrm{s}^{2}$ & $16.05 \mathrm{~km}^{2} / \mathrm{s}^{2}$ & $16.03 \mathrm{~km}^{2} / \mathrm{s}^{2}$ \\
\hline Venus & $21 \mathrm{April} 1998$ & $22 \mathrm{May} 1998$ & 29 April 1998 \\
\hline$\Delta v$ & $466 \mathrm{~m} / \mathrm{s}$ & $1974 \mathrm{~m} / \mathrm{s}$ & $431 \mathrm{~m} / \mathrm{s}$ \\
\hline Venus & $20 \mathrm{June} 1999$ & $01 \mathrm{July} 1999$ & 26 June 1999 \\
\hline$\Delta v$ & -- & $604 \mathrm{~m} / \mathrm{s}$ & -- \\
\hline Earth & 19 August 1999 & 20 August 1999 & 18 August 1999 \\
\hline$\Delta v$ & -- & $214 \mathrm{~m} / \mathrm{s}$ & -- \\
\hline Jupiter & 30 December 2000 & 01 January 2001 & 11 January 2001 \\
\hline$\Delta v$ & -- & -- & -- \\
\hline Saturn & $01 \mathrm{July} 2004$ & 04 June 2004 & $19 \mathrm{July} 2004$ \\
\hline$C_{3}$ (arrival) & $31.3 \mathrm{~km}^{2} / \mathrm{s}^{\wedge} 2$ & $29.9 \mathrm{~km}^{2} / \mathrm{s}^{2}$ & $26.8 \mathrm{~km}^{2} / \mathrm{s}^{2}$ \\
\hline
\end{tabular}


Table 3. Results for best-known solution to Cassini-like problem compared to grid optimization solutions in 1997 launch window. If a $\Delta v$ is not present on a given leg, a '..' is included in the table.

\begin{tabular}{|c|c|c|c|}
\hline Event & Best known & Grid Optimization & Refinement \\
\hline Launch & 13 November 1997 & 23 November 1997 & 11 November 1997 \\
\hline$C_{3}$ (departure) & $10.6 \mathrm{~km}^{2} / \mathrm{s}^{2}$ & $16.05 \mathrm{~km}^{2} / \mathrm{s}^{2}$ & $16.03 \mathrm{~km} / \mathrm{s}^{2}$ \\
\hline$\Delta v$ & $480 \mathrm{~m} / \mathrm{s}$ & -- & $462 \mathrm{~m} / \mathrm{s}$ \\
\hline Venus & 29 April 1998 & 20 May 1998 & 04 March 1998 \\
\hline$\Delta v$ & $398 \mathrm{~m} / \mathrm{s}$ & $1832 \mathrm{~m} / \mathrm{s}$ & $398 \mathrm{~m} / \mathrm{s}$ \\
\hline Venus & 27 June 1999 & 02 July 1999 & 28 June 1999 \\
\hline$\Delta v$ & -- & $682 \mathrm{~m} / \mathrm{s}$ & -- \\
\hline Earth & 20 August 1999 & 21 August 1999 & 20 August 1999 \\
\hline$\Delta v$ & -- & -- & -- \\
\hline Jupiter & 31 March 2001 & 18 April 2001 & 01 April 2001 \\
\hline$\Delta v$ & -- & -- & -- \\
\hline Saturn & 09 April 2007 & 24 October 2007 & 16 April 2007 \\
\hline$C_{3}$ (arrival) & $18.0 \mathrm{~km}^{2} / \mathrm{s}^{\wedge} 2$ & $17.7 \mathrm{~km}^{2} / \mathrm{s}^{2}$ & $18.0 \mathrm{~km}^{2} / \mathrm{s}^{2}$ \\
\hline
\end{tabular}

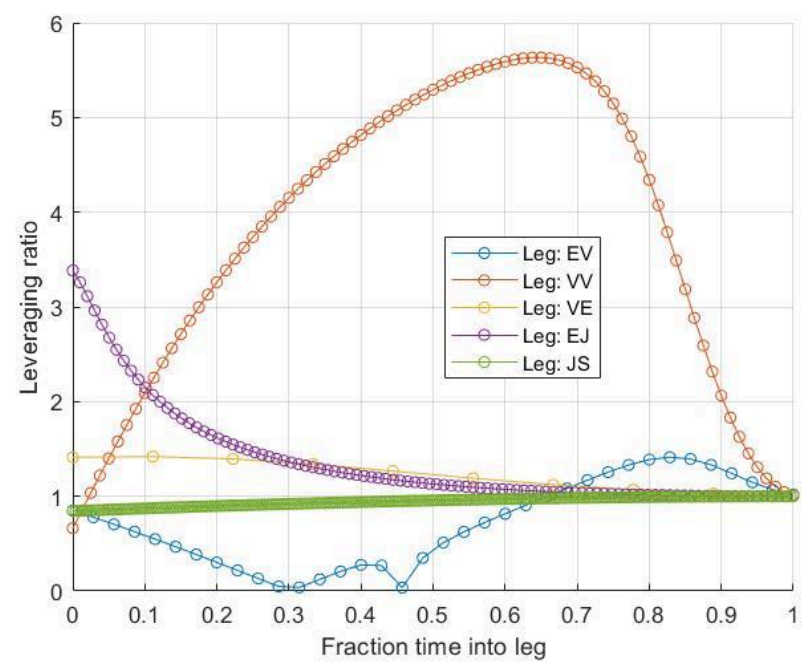

Fig. 10 Leveraging ratios per leg considered for actual Cassini mission.

When comparing the fully optimized solution coming from the refinement step with the first grid-based solution, in the full solutions the defect at the start of the second leg (i.e., on the Venus-Venus one) has been removed by lowering the infinity velocity at Venus, with no DSM applied in the first leg. This lower initial infinity velocity at Venus causes a large defect at the second Venus encounter, but it is corrected by a DSM in the second leg, where high efficiency of DSMs is seen. The grid-based solution has higher cost (i.e., $\Delta v$ prediction) than the optimised solutions, because the optimization performed in the refinement step redistributes the infinity velocity defects to maximally utilise the DSM leveraging. This is also noticed from the leveraging ratios computed on each leg of the EVVEJS transfer in Fig. 10. The parameter plotted is the ratio of the infinity velocity defect corrected to magnitude of a DSM versus time elapsed into the segment. Leveraging rations are obtained through the procedure described in 6 . The plot illustrates that in the second segment (i.e., the Venus-Venus leg), the peak ratio is close to 6, and therefore it implies that any infinity velocity defect can be efficiently corrected with a much smaller DSM, which is what happens in the refinement. In the first segment (Earth-Venus), the ratio is generally less than 1, but it exceeds 1 towards the end of the leg, reaching approximately 1.4. The efficiency in this segment is clearly much less than the Venus-Venus one. It is also interesting to notice that in the last Jupiter-Saturn leg, the leveraging ratio remains below 1 for all the time elapsed in the segment, reaching its maximum value i.e., 1, only at Saturn. This suggests that no DSM are useful to leverage the infinity velocity at Saturn, as also confirmed by optimized results in Table 2. 


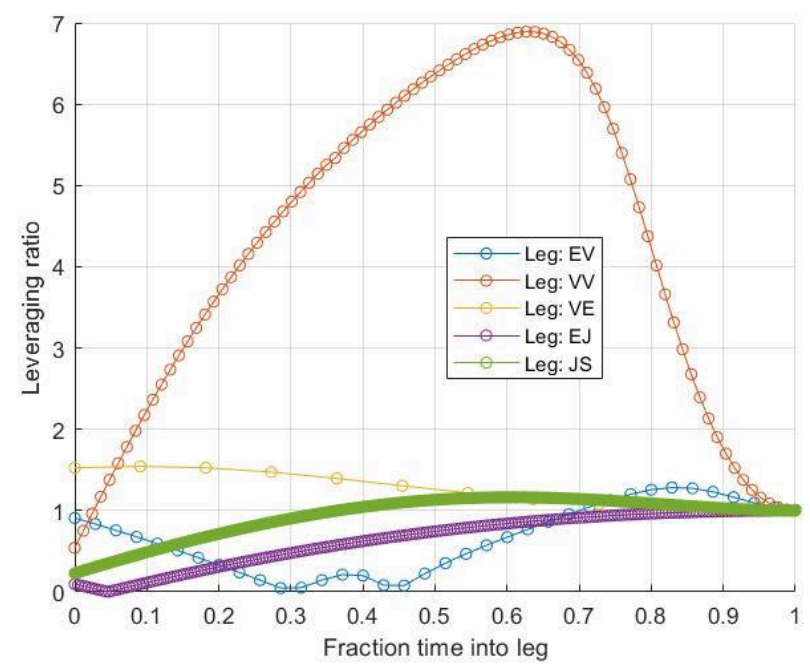

Fig. 11 Leveraging ratios per leg considered for global optimum solution of EVVEJS mission.

The nominal Cassini mission transfer towards Saturn lasts under 7 years from launch to rendezvous with Saturn. As a fast and time-constrained transfer, this mission does not represent the optimum. Faster transfers increase the infinity velocity at Saturn and lead to increased infinity velocity at Earth and larger DSMs. The best reported solution for the Saturn problem at this launch years, as compared to the actual Cassini one, is characterized by lower infinity velocities at departure and arrival, but with much higher transfer time (mainly due to the last Jupiter-Saturn leg of the transfer). However, this is still constrained in terms of duration. The Pareto set shown in Fig. 7 includes constraints on the legs' duration. To allow a search for global optimum, these should be released, as one might expect the actual global minimum solution to lie much farther to the right in terms of transfer times. In fact, when duration constraints are removed and therefore the total mission duration extends, a significant improvement in objective can be obtained. This is confirmed from Table 4 describing results on which a much larger transfer time is employed for the last JupiterSaturn leg. Regarding the grid optimization solution, the transfer duration is about 17.3 years, with an objective value of $9.50 \mathrm{~km} / \mathrm{s}$. This is not far from best solution obtained with constrained total time of flight of about 9.9 years, on which $10.6 \mathrm{~km} / \mathrm{s}$. However, when refined, global optimum solution is significantly better in terms of $\Delta v$ with respect to refined best-known solution (see again Table 3). Part of this is the removal of the infinity velocity defects and replacement with DSMs in the early transfer legs. However, there is also a significant effect on the last leg. From Fig. 11 , by evaluating the leveraging ratio on the last Jupiter-Saturn segment, one notices that it exceeds 1 around the midregion of the last leg. Therefore, it is more efficient to remove most of the final infinity velocity at Saturn with a large DSM between Jupiter and Saturn. However, by applying this leveraging DSM, a large time increment in the arrival time is needed (in the order of 12 years), which is confirmed by the refined results, where usually the correction required is in the order of few days. This is also noticeable from Fig. 12, representing trajectories on the ecliptic plane before and after the refinement when time constraints on the last leg are removed.

A general consideration is that, when large part of the total cost for the mission consists in infinity velocity defects, then PSO optimization scales down the solutions by replacing defects with optimal DSMs. Grid-based optimization will give the most accurate predictions for near-ballistic transfers with zero or low DSM need and shows greater divergence from fully optimized solutions where large DSMs are needed. The DSM at about $400 \mathrm{~m} / \mathrm{s}$ in the early trajectory phase of the Cassini transfer, for example, is relatively large when compared with many interplanetary transfers. However, the use of grid-based optimization to initialize the subsequent refinement leads to the expected optimal solution. 

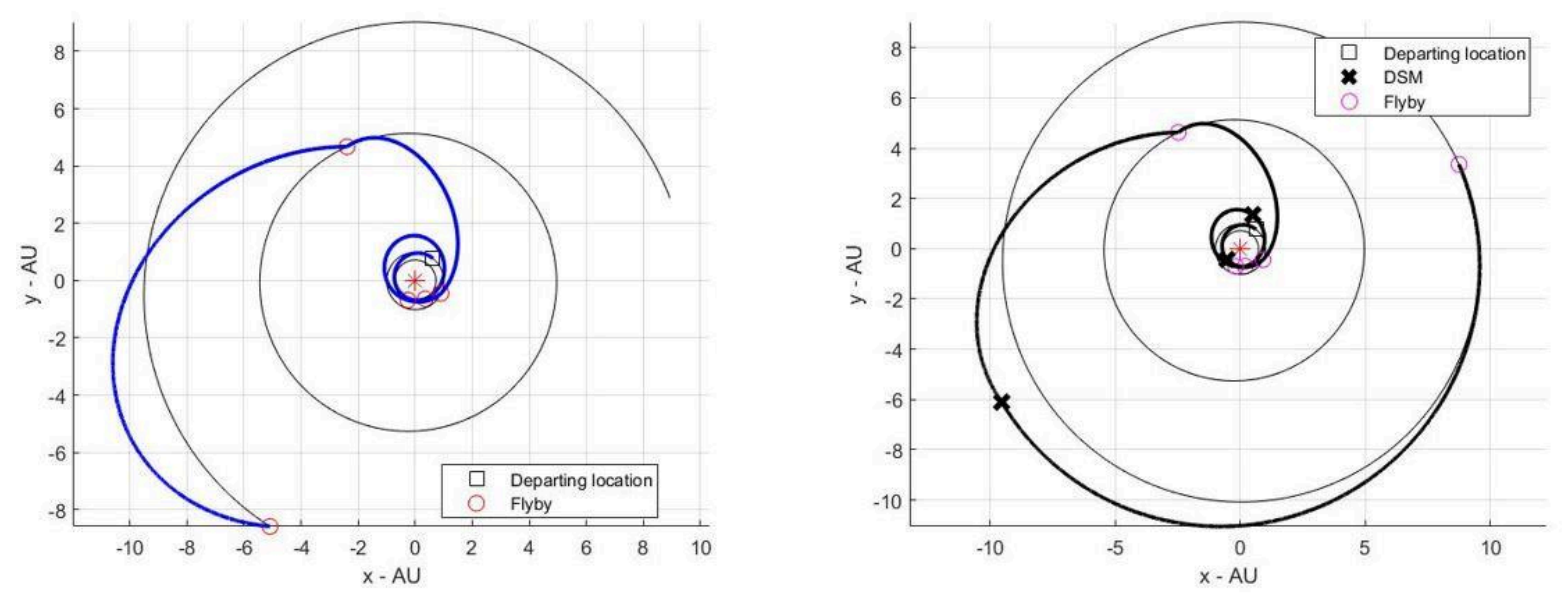

Fig. 12 Optimum solution for EVVEJS transfer with departure date in 1997. Left plot is obtained with defects formulation, while right plot with DSMs formulation. From innermost to outermost, black circles represent Venus, Earth, Jupiter, and Saturn orbits, respectively.

Table 4. Results for optimum solution to Cassini-like problem in 1997 launch window. If a $\Delta \mathrm{v}$ is not present on a given leg, a '--', is included in the table.

\begin{tabular}{|c|c|c|}
\hline Event & Grid Optimization & Refinement \\
\hline Launch & 13 November 1997 & 13 November 1997 \\
\hline$C_{3}$ (departure) & $13.2 \mathrm{~km}^{2} / \mathrm{s}^{2}$ & $11.1 \mathrm{~km}^{2} / \mathrm{s}^{2}$ \\
\hline$\Delta v$ & -- & $394 \mathrm{~m} / \mathrm{s}$ \\
\hline Venus & 10 May 1998 & $02 \mathrm{May} 1998$ \\
\hline$\Delta v$ & $1419 \mathrm{~m} / \mathrm{s}$ & $195 \mathrm{~m} / \mathrm{s}$ \\
\hline Venus & 02 July 1999 & 07 July 1999 \\
\hline$\Delta v$ & $123 \mathrm{~m} / \mathrm{s}$ & -- \\
\hline Earth & 27 August 1999 & 28 August 1999 \\
\hline$\Delta v$ & $52 \mathrm{~m} / \mathrm{s}$ & -- \\
\hline Jupiter & 18 July 2002 & 31 July 2002 \\
\hline$\Delta v$ & -- & $2273 \mathrm{~m} / \mathrm{s}$ \\
\hline Saturn & $02 \mathrm{March} 2015$ & 16 July 2027 \\
\hline$C_{3}$ (arrival) & $18.2 \mathrm{~km}^{2} / \mathrm{s}^{2}$ & $0.248 \mathrm{~km}^{2} / \mathrm{s}^{2}$ \\
\hline
\end{tabular}

\section{E. Sample Return Missions Towards Comets}

The first family explored for sample return mission options is the one of Jupiter Family Comets (JFCs). These objects are characterized by periapsis of approximately $1 \mathrm{AU}$ and apoapsis close to the orbit of Jupiter, which makes them accessible for sample return and rendezvous opportunities. A mission to those comets is made by a cruise phase needed to reach the desired object, an operational phase, on which the spacecraft position and velocity are assumed to match the ones of the comet for sampling purposes, and another cruise phase for returning the cometary samples to the Earth. From Fig. 13, representing JFCs orbits on Tisserand map, some JFCs can be accessed with virtually zero $\Delta v$ if Mars, Earth of Jupiter are considered for a last planetary encounter. This is the case of the comet 67P/Churyumov-Gerasimenko, i.e. the target of Rosetta mission [27], on which Mars can be considered as last fly-by planet to rendezvous with the comet at null cost. For the present work, sample return options for 67P are investigated, although with the procedure described in section 7 a full catalogue of options towards JFCs is obtainable. 


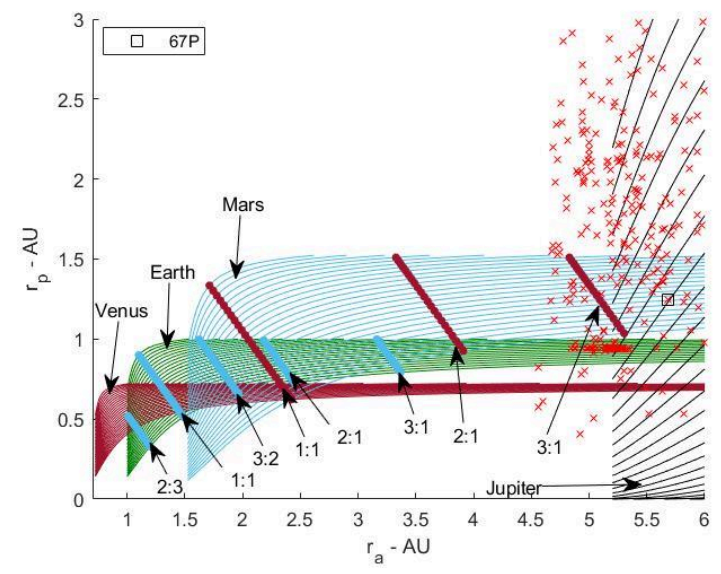

Fig. 13 Tisserand map representing Venus, Earth, Mars, and Jupiter contours with infinity velocities ranging from 3 to $15 \mathrm{~km} / \mathrm{s}$ spaced by $0.5 \mathrm{~km} / \mathrm{s}$. 2:3, 1:1, 3:2, 2:1 and 3:1 resonant orbits for Earth and 1:1, 2:1 and 3:1 resonant orbits for Mars are represented. Apoapsis and periapsis of JFCs are also reported.

However, due to the high apoapsis of 67P, several Mars fly-by would be required, involving 2:1 and 3:1 resonant orbits which imply long transfer times, resulting in unfeasible mission options, especially if a sample return is considered. Therefore, fly-bys with Earth and Venus are also considered to speed up the overall transfer, due to higher planetary masses. Tisserand map exploration in fact shows that sequences like EVEE, EMEE (the one employed by Rosetta), EVVE, EVEM and EMEM are in fact able to reach 67P orbit. From Tisserand map exploration, one should notice that if Earth is considered as last planetary encounter, compared to Mars encounter, a higher $\Delta v$ is needed to rendezvous with the comet, to increase the periapsis of spacecraft orbit to the one of 67P.

When optimized with grid-based optimization as described in section 5, Pareto fronts can be obtained as shown in Fig. 14, on which only few of those sequences appear feasible in the selected time window. The operations phase at cometary rendezvous are assumed to take place between 6 to 12 months after the comet arrival. One notices that, for the instance considered, the well-known EVEE and EVEM sequences perform well for reaching the comet, while EEME and EEVE share very similar performances when considering the return phase. It is worth noticing that, even though from Tisserand map exploration Mars results in being the best planet to reach the comet orbit, when looking for optimal trajectories on a full planetary ephemerides model, sequences involving Earth and Venus results in being the optimal ones. This is because the synchronicity between Earth and Venus for cheap transfers is more likely to happen when compared to Mars, resulting in more expensive transfers overall.
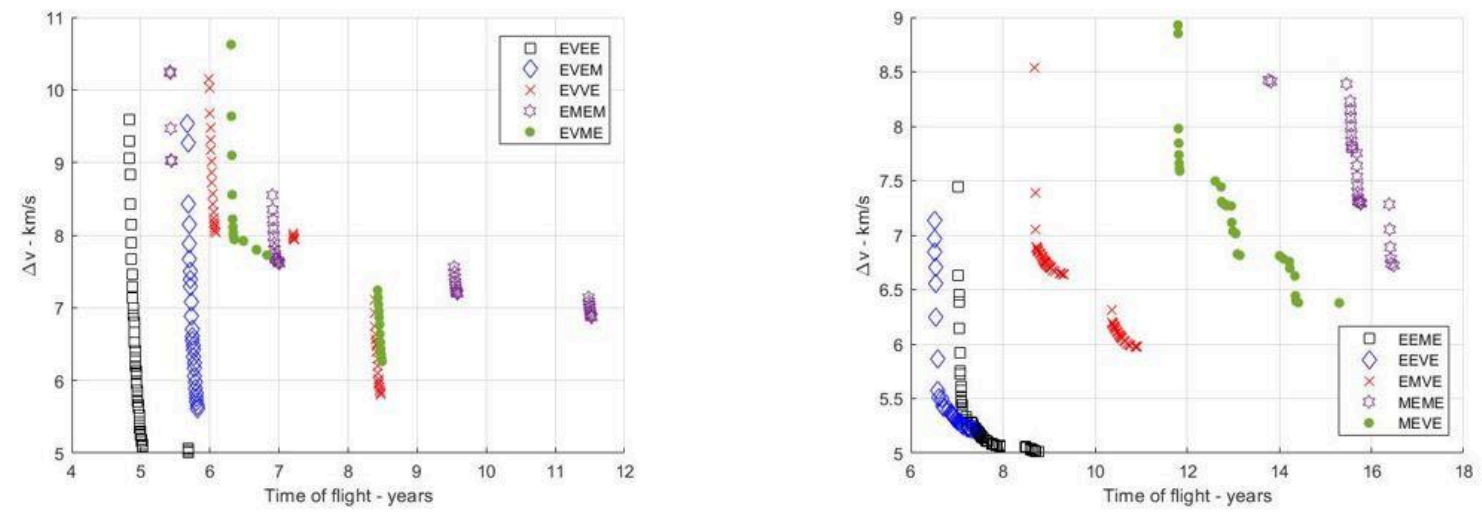

Fig. 14 Pareto fronts for missions towards 67P (left) and return (right) for an arrival to the comet in the 20352045 timeframe as from grid-based optimization. 
Table 5. Sample return mission towards 67P as coming from grid optimization involving EVEM and EEVE sequences for cometary rendezvous and return phases, respectively. If a $\Delta v$ is not present on a given leg, a '--', is included in the table.

\begin{tabular}{|c|c|c|c|}
\hline \multicolumn{2}{|c|}{ Towards the comet } & \multicolumn{2}{c|}{ Return phase } \\
\hline Event & Value & Event & Value \\
\hline Launch & 2 June 2039 & Launch & 24 September 2045 \\
\hline$C_{3}$ (departure) & $10.34 \mathrm{~km}^{2} / \mathrm{s}^{2}$ & $\Delta v$ (Comet departure) & $1028.2 \mathrm{~m} / \mathrm{s}$ \\
\hline$\Delta v$ & -- & $\Delta v$ & -- \\
\hline Venus & 19 November 2039 & Earth & 7 November 2047 \\
\hline$\Delta v$ & -- & $\Delta v$ & -- \\
\hline Earth & 15 October 2041 & Earth & 7 November 2049 \\
\hline$\Delta v$ & $1034.4 \mathrm{~m} / \mathrm{s}$ & $\Delta v$ & $289.1 \mathrm{~m} / \mathrm{s}$ \\
\hline Mars & 22 February 2042 & Venus & 12 March 2052 \\
\hline$\Delta v$ & $414.6 \mathrm{~m} / \mathrm{s}$ & $\Delta v$ & -- \\
\hline $67 \mathrm{P}$ & $28 \mathrm{March} 2045$ & Earth & 21 August 2052 \\
\hline$\Delta v$ (Comet rendezvous) & $944.5 \mathrm{~m} / \mathrm{s}$ & $C_{3}$ (arrival) & $15.92 \mathrm{~km}^{\mathrm{s}} / \mathrm{s}^{2}$ \\
\hline
\end{tabular}

Table 6. Refined EVEM sequence towards 67P. If a $\Delta v$ is not present on a given leg, a '--', is included in the table.

\begin{tabular}{|c|c|}
\hline \multicolumn{2}{|c|}{ Towards the comet } \\
\hline Event & Value \\
\hline Launch & 4 June 2039 \\
\hline$C_{3}$ (departure) & $10.55 \mathrm{~km}^{2} / \mathrm{s}^{2}$ \\
\hline$\Delta v$ & -- \\
\hline Venus & 19 November 2039 \\
\hline$\Delta v$ & -- \\
\hline Earth & 13 October 2041 \\
\hline$\Delta v$ & $1017.2 \mathrm{~m} / \mathrm{s}$ \\
\hline Mars & 21 February 2042 \\
\hline$\Delta v$ & $431.1 \mathrm{~m} / \mathrm{s}$ \\
\hline $67 \mathrm{P}$ & $23 \mathrm{March} 2045$ \\
\hline$\Delta v$ (Comet rendezvous) & $868.0 \mathrm{~m} / \mathrm{s}$ \\
\hline
\end{tabular}
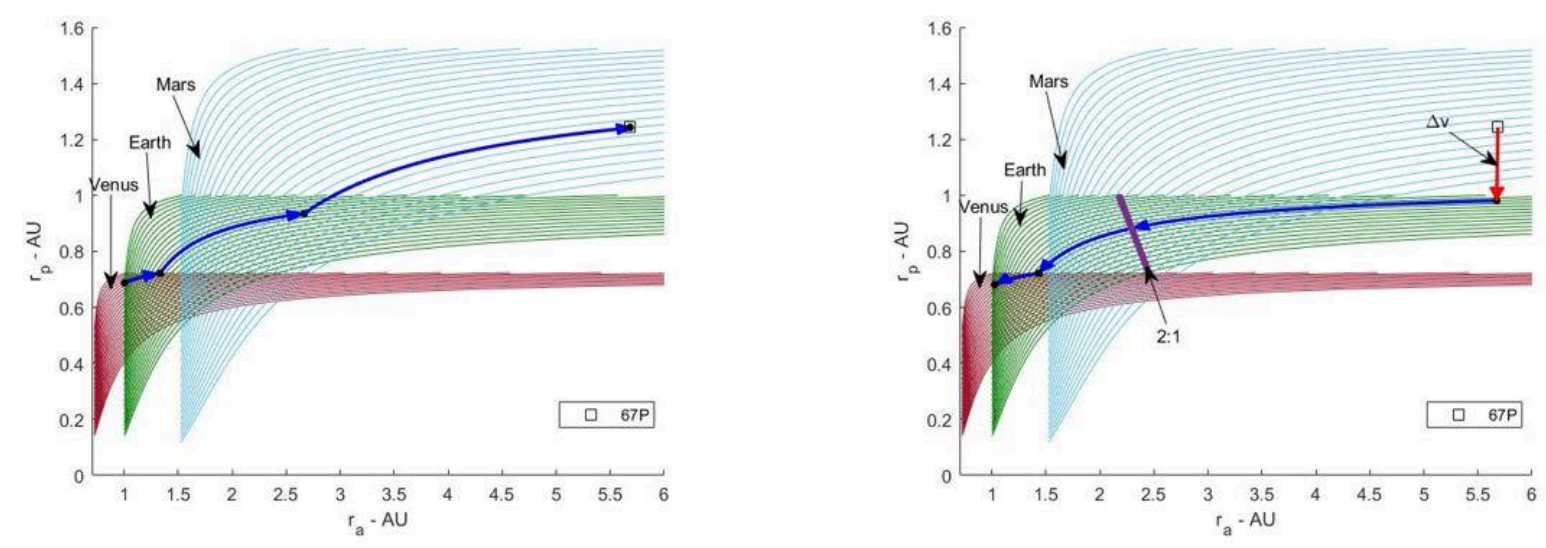

Fig. 15 EVEM (left) and (EEVE) sequences for 67P sample return as resulting from Tisserand map exploration. The red arrow represents the $\Delta v$ needed to reach 67P after last Earth fly-by. 2:1 Earth resonant orbits are also represented. Venus, Earth, and Mars contours are also reported with infinity velocities ranging from 3 to $15 \mathrm{~km} / \mathrm{s}$ spaced by $0.5 \mathrm{~km} / \mathrm{s}$.

This is also confirmed in Table 5 and Table 6 showing an example of full sample return mission towards 67P involving EVEM and EEVE transfers for the two phases of the mission (see also Fig. 16 and Fig. 17 for trajectories 
representation). Note that Table 6 and Fig. 17 only reports optimized solution for EVEM sequence, as the EEVE one does not vary much from the grid optimization. From Fig. 15, as expected from Tisserand map exploration, having Mars as last fly-by body allows to have low $\Delta v$ for cometary rendezvous, but high defects to encounter the planet, due to rare synchronicity with other planets. On the other hand, having the Earth as last fly-by encounter permits quite lower defects during the swing-by sequence, but with substantial $\Delta v$ for departing 67P.
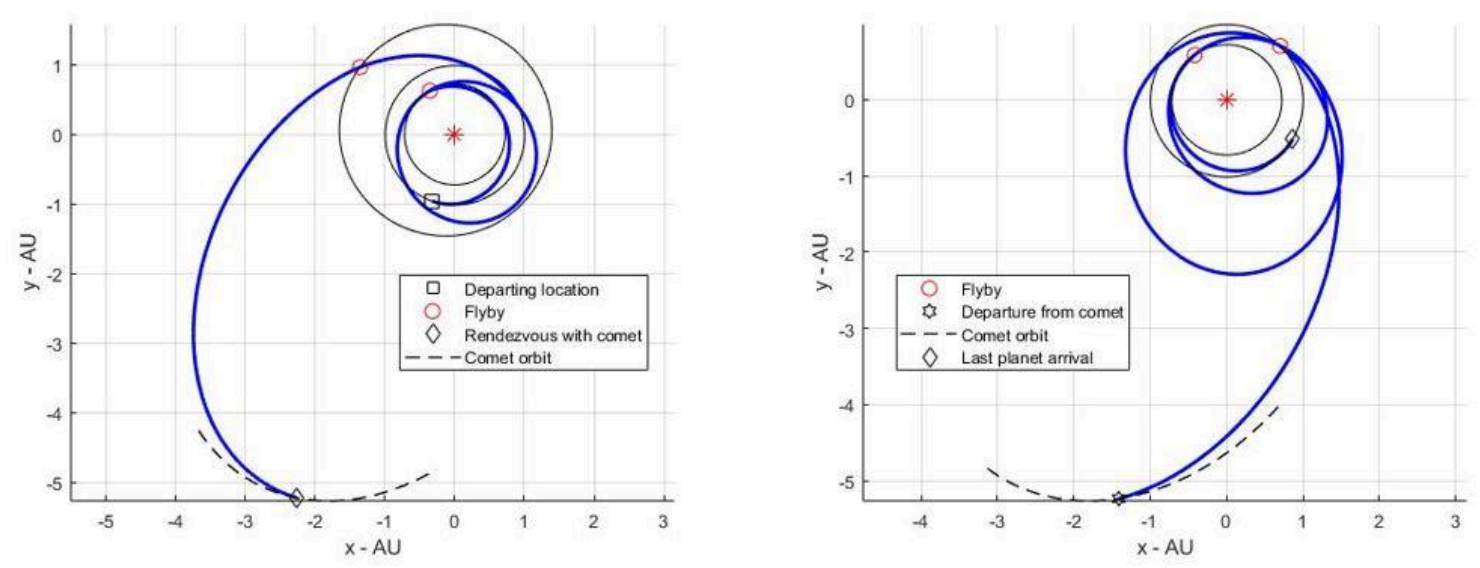

Fig. 16 EVEM (left) and EEVE (right) transfers for 67P sample return as from grid optimization. From innermost to outermost, black circles represent Venus, Earth, and Mars orbits (left plot), respectively, and Venus and Earth orbits (right plot), respectively.

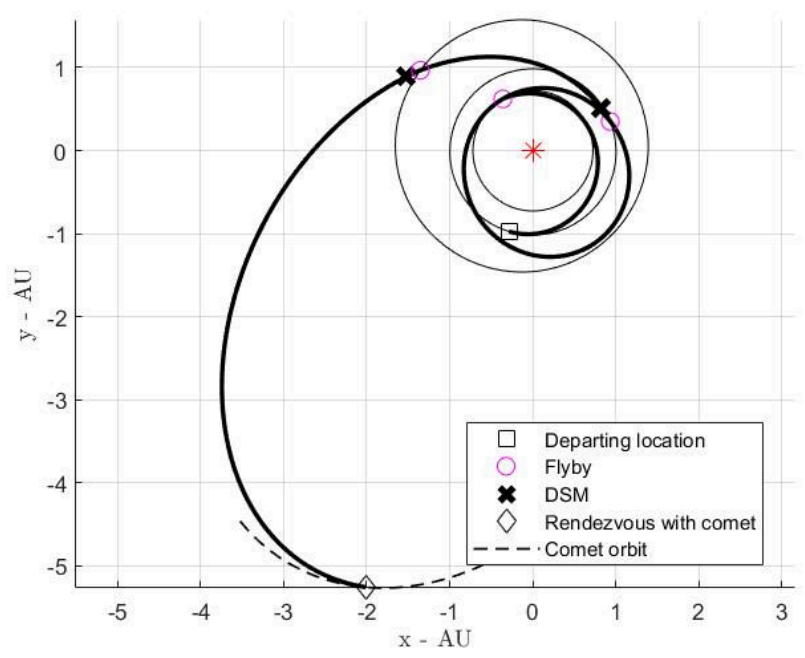

Fig. 17 Illustration of refined EVEM sequence towards 67P. From innermost to outermost, black circles represent Venus, Earth, and Mars orbits.

Another interesting case for rendezvous missions is represented by Centaurs, which are objects with wide range in orbits, from nearly circular ones, right outside Jupiter orbit, to those ones beyond Uranus, as well as those with eccentricity that crosses planets orbits. Typical classification [28] of these objects considers them to have perihelion and semi-major axis between the orbits of Jupiter and Neptune. Fig. 18 illustrates eccentricity, inclination, and semimajor axis of known Centaurs. 


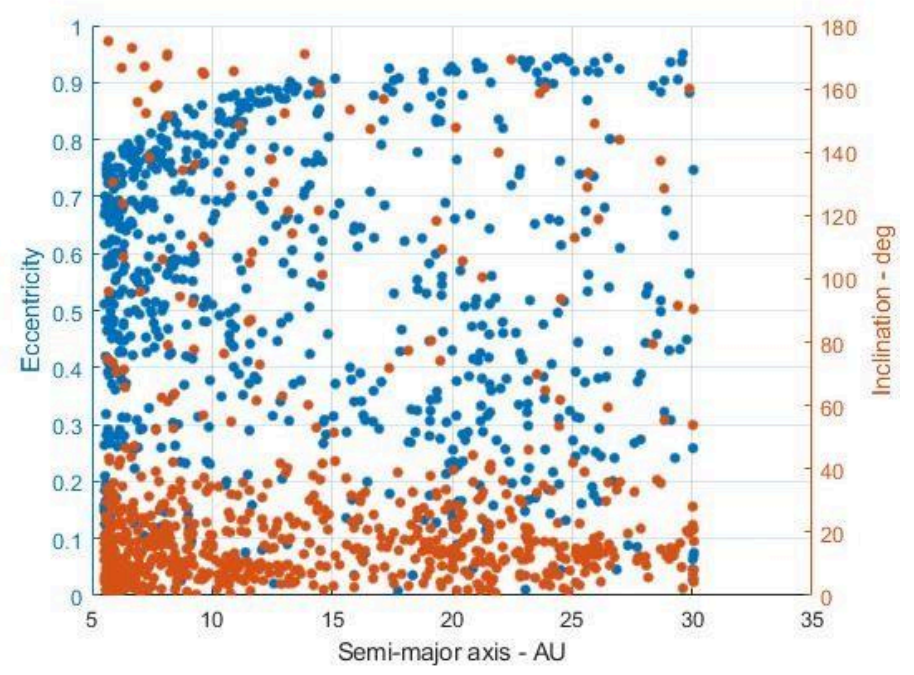

Fig. 18 Eccentricity, inclination, and semi-major axis for Centaur objects.

A potential rendezvous mission to Centaurs is of interest to understand comet activity going beyond the water ice snowline, as well as to observe early phases of in cometary evolution. Moreover, as from Fig. 18, most objects are characterized by relatively high inclination with respect to the ecliptic plane, thus providing the chance to observe the Solar System from a privileged point of view. Therefore, for the purposes of the present paper, a Centaur object with high inclination (i.e., greater than $40 \mathrm{deg}$ ) is selected as target, namely 2017/R17, to explore potential rendezvous opportunities as reference for future missions' options. Table 7 reports main Keplerian orbital elements for 2017/R17.

Table 7. Keplerian elements for Centaur object 2017/R17.

\begin{tabular}{|c|c|}
\hline Keplerian element & Value \\
\hline Semi-major axis & $6.62 \mathrm{AU}$ \\
\hline Eccentricity & 0.45 \\
\hline Inclination & $42.69 \mathrm{deg}$ \\
\hline Right Ascension of the Ascending Node & $135.67 \mathrm{deg}$ \\
\hline Argument of Periapsis & $295.53 \mathrm{deg}$ \\
\hline
\end{tabular}

Transfers to such objects are characterized by long transfer times, as well as by relatively high $\Delta v$ for plane change manoeuvres and rendezvous. To achieve such inclinations, a close passage with Jupiter is considered as last planetary fly-by. The high mass of Jupiter allows to perform the plane change without spending any $\Delta v$. Moreover, to further mitigate the impact of 2017/R17 orbit on trajectory design, the last planetary encounter shall occur close to the orbital nodes of the Centaur, i.e., at about $135.67 \mathrm{deg}$ and $315.67 \mathrm{deg}$ of solar longitude. A tolerance of $\pm 5 \mathrm{deg}$ is employed. Fig. 19 reports Pareto fronts for a rendezvous mission towards 2017/R17 for different sequences departing in the 2040-2045 timeframe. As it can be seen, a whole range of options appear, with a clear trend of decreasing $\Delta v$ at higher transfer times. As expected, favorable options in terms of $\Delta v$ exist at very high cruise duration, while very few show up in a feasible range of propellant consumption and transfer times. The latter belong to the well-known EVEEJ sequence with 2:1 resonance on the successive Earth fly-bys, which performs well for the selected scenario, showing a Pareto front that dominates all the other solutions. As transfer times increase, other planetary sequences might become competitive such as EVVEJ. Sequences employing Mars fly-bys do not seem competitive, and this is mainly because of the rare synchronicity between Mars with other planetary encounters. For example, the sequence EVEMEJ, i.e. the same one selected as baseline transfer for JUICE mission [29], repeats with similar performances with a period of about 12 years, while sequences involving only Earth, Venus and Jupiter occur every 1-2 years. Fig. 20 and Fig. 21 represents fast and long 3D trajectories for EVEEJ-2017/R17 transfers, respectively. Fast transfer refers to a solution from Pareto fronts in Fig. 18 with $<8$ years and $\Delta v<8.5 \mathrm{~km} / \mathrm{s}$, considered feasible in context of practical mission design, while the long transfer is the top right solution of the same Pareto set. Table 8 and Table 9 provide details of the two solutions, respectively, both for the grid optimization and the post-processing. The main difference between fast and short transfer relies on the presence of DSMs as well as in an increased $\Delta v$ required for the manoeuvre. In the case of fast mission, it is worth noticing that defects on Venus-Earth and Earth-Jupiter legs are 
mapped almost unmodified in the successive optimization, to reduce the next defect, i.e., the one on the JupiterCentaur leg, to zero. The overall fast trajectory as coming from the refinement optimization is thus cheaper in terms of $\Delta v$, while the time-of-flight results slightly increased, i.e., from about 7.798 years to 8.238 years. On the other hand, the long transfer option maps quite well from the grid optimization to the refinement stage, as defects from grids optimization were already quite small. This again proves that ballistic transfers are easily transcribed from grid to PSO refinement.

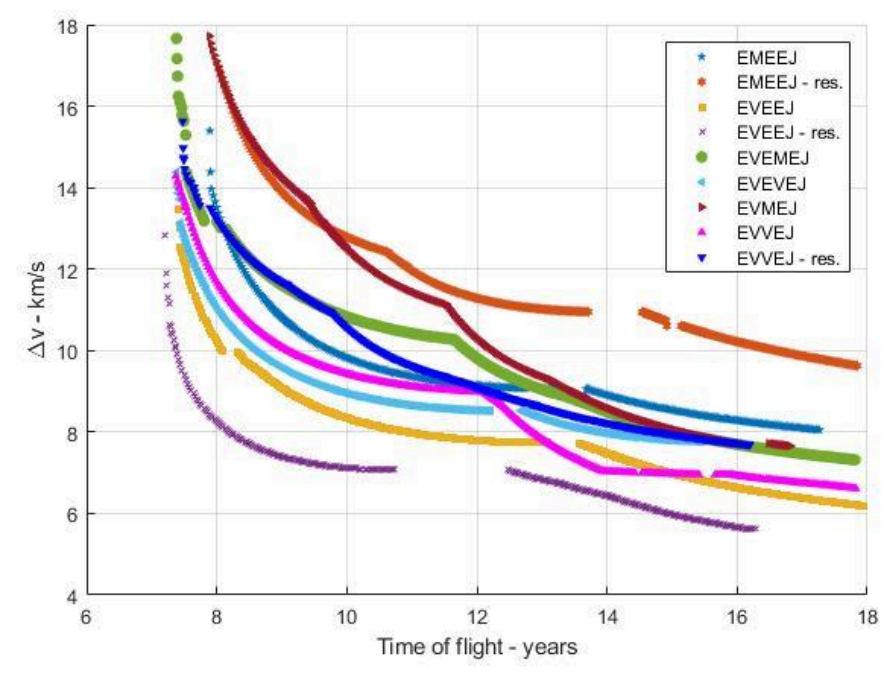

Fig. 19 Pareto front of some sequences for rendezvous with 2017/R17 departing in the 2040-2045 timeframe. In the legend, when 'res' is present, a 2:1 resonance is used on the Earth-Earth fly-by.
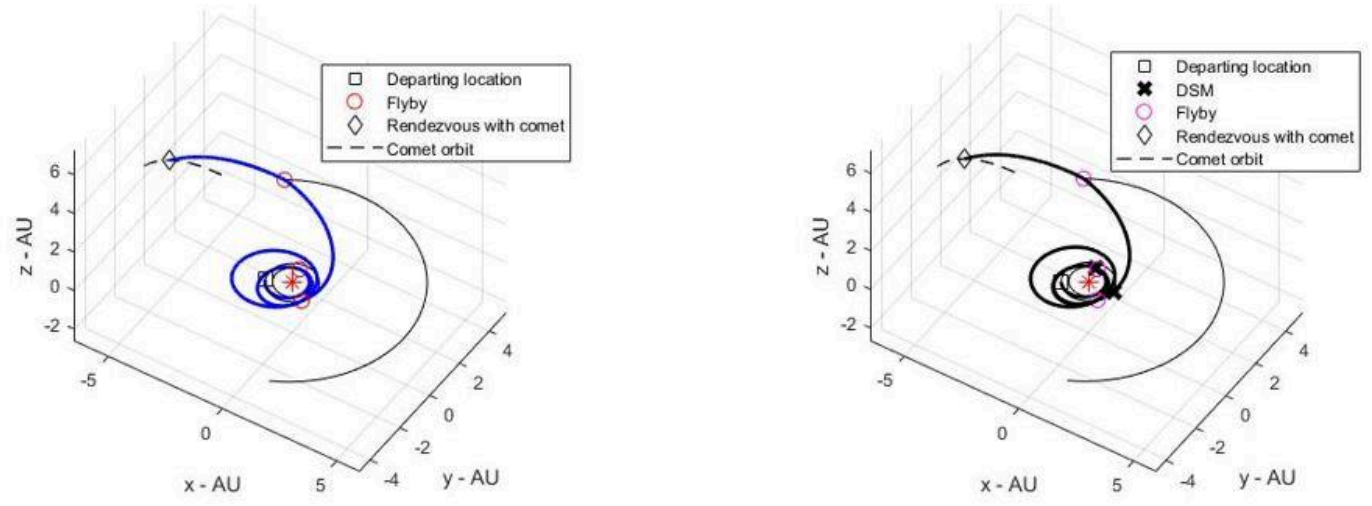

Fig. 20 3D representation of a fast transfer to 2017/R1 employing an EVEEJ sequence. Left plot is obtained with defects formulation, while right plot with DSMs formulation. From innermost to outermost, black circles represent Venus, Earth, and Jupiter orbits, respectively. 

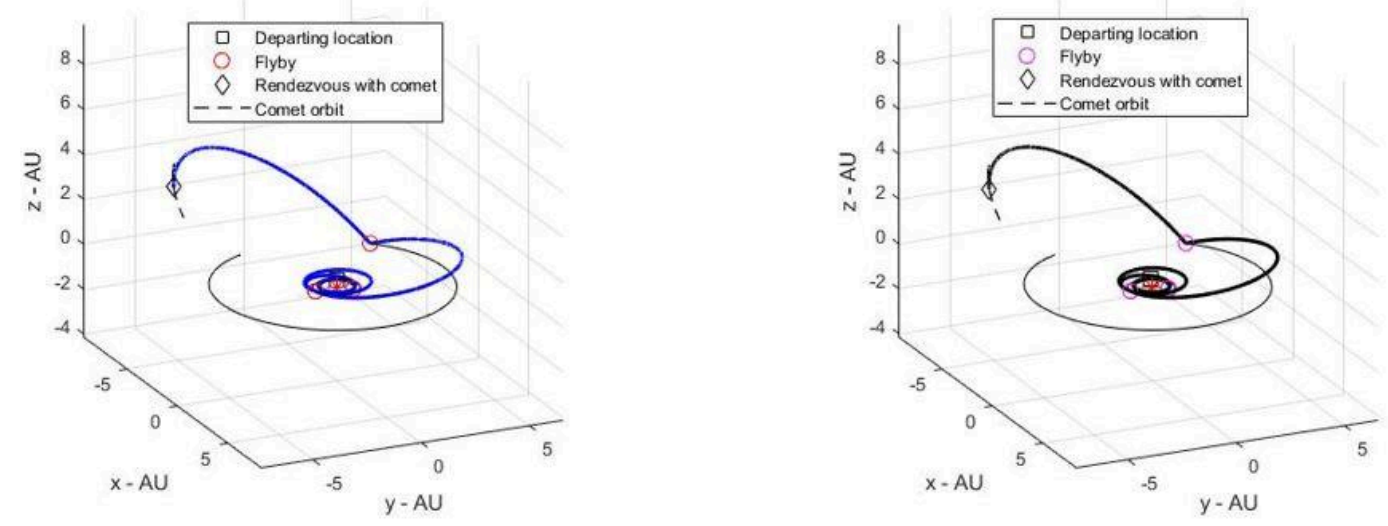

Fig. 21 3D representation of a long transfer to 2017/R1 employing an EVEEJ sequence. Left plot is obtained with defects formulation, while right plot with DSMs formulation. From innermost to outermost, black circles represent Venus, Earth, and Jupiter orbits, respectively.

Table 8. Results for fast EVEEJ transfer to 2017/R17 in 2040-2045 launch window. If a $\Delta v$ is not present on a given leg, a '--', is included in the table.

\begin{tabular}{|c|c|c|}
\hline Event & Grid Optimization & Refinement \\
\hline Launch & 14 April 2044 & 23 April 2044 \\
\hline$C_{3}$ (departure) & $13.12 \mathrm{~km}^{2} / \mathrm{s}^{2}$ & $13.12 \mathrm{~km}^{2} / \mathrm{s}^{2}$ \\
\hline$\Delta v$ & -- & -- \\
\hline Venus & 9 October 2044 & 5 October 2044 \\
\hline$\Delta v$ & $24.2 \mathrm{~m} / \mathrm{s}$ & $24.9 \mathrm{~m} / \mathrm{s}$ \\
\hline Earth & 16 August 2046 & 14 August 2046 \\
\hline$\Delta v$ & -- & 13 August 2048 \\
\hline Earth & 16 August 2048 & $797.5 \mathrm{~m} / \mathrm{s}$ \\
\hline$\Delta v$ & $747.4 \mathrm{~m} / \mathrm{s}$ & 28 March 2050 \\
\hline Jupiter & 11 April 2050 & -- \\
\hline$\Delta v$ & $521.1 \mathrm{~m} / \mathrm{s}$ & 19 February 2052 \\
\hline $2017 /$ R17 & 31 January 2052 & $3569.0 \mathrm{~mm} / \mathrm{s}$ \\
\hline$\Delta v$ (rendezvous) & $3668.0 \mathrm{~m} / \mathrm{s}$ & \\
\hline
\end{tabular}

Table 9. Results for long EVEEJ transfer to 2017/R17 in 2040-2045 launch window. If a $\Delta v$ is not present on a given leg, a '--', is included in the table.

\begin{tabular}{|c|c|c|}
\hline Event & Grid Optimization & Refinement \\
\hline Launch & 24 February 2041 & 25 February 2041 \\
\hline$C_{3}$ (departure) & $15.56 \mathrm{~km}^{\mathrm{s}} / \mathrm{s}^{2}$ & $15.68 \mathrm{~km}^{2} / \mathrm{s}^{2}$ \\
\hline$\Delta v$ & -- & -- \\
\hline Venus & 13 August 2041 & 13 August 2041 \\
\hline$\Delta v$ & $16.6 \mathrm{~m} / \mathrm{s}$ & -- \\
\hline Earth & 25 June 2042 & 25 June 2042 \\
\hline$\Delta v$ & -- & -- \\
\hline Earth & 25 June 2044 & 24 June 2044 \\
\hline$\Delta v$ & $72.9 \mathrm{~m} / \mathrm{s}$ & -- \\
\hline Jupiter & 29 September 2050 & 25 September 2050 \\
\hline$\Delta v$ & $18.5 \mathrm{~m} / \mathrm{s}$ & -- \\
\hline $2017 / \mathrm{R} 17$ & 4 July 2057 & 2 July 2057 \\
\hline
\end{tabular}




\begin{tabular}{|c|c|c|}
\hline$\Delta v$ (rendezvous) & $1575.1 \mathrm{~m} / \mathrm{s}$ & $1566.0 \mathrm{~m} / \mathrm{s}$ \\
\hline
\end{tabular}

\section{Conclusion}

This paper presented an innovative approach based upon the Bellman's principle of optimality and dynamic programming to automatically design long MGA trajectories to cometary bodies, employing high number of planetary encounters, more than any mission considered before. The key aspect of the methodology consists in approximating the required DSMs as infinity velocity defects at each fly-by, to generate wide sets of solutions to represent broad Pareto sets, useful for preliminary mission analysis. This enables a reduction of the number of design parameters, while maintaining a good representation of the search space. The understanding of the effectiveness of this approach is further enhanced by the evaluation of the relationship between impulse model types.

Moreover, preliminary results for sample return and rendezvous missions for cometary objects and never-explored bodies and orbital regions are presented, to prove the effectiveness of the proposed methods.

Future work will concentrate on providing full missions scenarios and launch windows analysis for both comet sample return and high-inclined Centaurs objects, both with impulsive and low-thrust transfers. Moreover, additional research will be conducted to focus on mapping swing-by discontinuities into actual DSMs while in the search step, maintaining efficient computational efforts.

\section{Acknowledgments}

This work was supported by Airbus Defense and Space through the Cranfield University Industrial Partnership Framework (MITnTargets: Mixed-Integer Trajectory Design for Large Number of Targets).

\section{References}

[1] R. Reinhard, The Giotto encounter with comet Halley, Nature. 321 (1986) 313-318.

[2] M.D. Rayman, P. Varghese, D.H. Lehman, L.L. Livesay, Results from the Deep Space 1 technology validation mission, Acta Astronaut. 47 (2000) 475-487.

[3] D. Brownlee, The Stardust mission: analyzing samples from the edge of the solar system, Annu. Rev. Earth Planet. Sci. 42 (2014) 179-205.

[4] W.H. Blume, Deep impact mission design, Space Sci. Rev. 117 (2005) 23-42.

[5] K.-H. Glassmeier, H. Boehnhardt, D. Koschny, E. Kührt, I. Richter, The Rosetta mission: flying towards the origin of the solar system, Space Sci. Rev. 128 (2007) 1-21.

[6] D. Bockelée-Morvan, G. Filacchione, K. Altwegg, E. Bianchi, M. Bizzarro, J. Blum, L. Bonal, F. Capaccioni, M. Choukroun, C. Codella, AMBITION-comet nucleus cryogenic sample return, Exp. Astron. (2021) 1-52.

[7] C.M. Chilan, B.A. Conway, A space mission automaton using hybrid optimal control, in: 17th Annu. Sp. Flight Mech. Meet., 2007: pp. 259-276.

[8] M. Ceriotti, M. Vasile, Automated multigravity assist trajectory planning with a modified ant colony algorithm, J. Aerosp. Comput. Information, Commun. 7 (2010) 261-293.

[9] A. Gad, O. Abdelkhalik, Hidden genes genetic algorithm for multi-gravity-assist trajectories optimization, J. Spacecr. Rockets. 48 (2011) 629-641.

[10] M. Schlueter, S.O. Erb, M. Gerdts, S. Kemble, J.-J. Rückmann, MIDACO on MINLP space applications, Adv. Sp. Res. 51 (2013) 1116-1131.

[11] N.J. Strange, J.M. Longuski, Graphical method for gravity-assist trajectory design, J. Spacecr. Rockets. 39 (2002) 9-16.

[12] A.F. Heaton, N.J. Strange, J.M. Longuski, E.P. Bonfiglio, Automated design of the Europa Orbiter tour, J. Spacecr. Rockets. 39 (2002) 17-22.

[13] K.W. Kloster, A.E. Petropoulos, J.M. Longuski, Europa Orbiter tour design with Io gravity assists, Acta Astronaut. 68 (2011) 931-946.

[14] S. Campagnola, B.B. Buffington, A.E. Petropoulos, Jovian tour design for orbiter and lander missions to Europa, Acta Astronaut. 100 (2014) 68-81.

[15] G. Colasurdo, A. Zavoli, A. Longo, L. Casalino, F. Simeoni, Tour of Jupiter Galilean moons: Winning solution of GTOC6, Acta Astronaut. 102 (2014) 190-199.

[16] S. Campagnola, R.P. Russell, Endgame problem part 1: V-infinity-leveraging technique and the leveraging graph, J. Guid. Control. Dyn. 33 (2010) 463-475.

[17] S. Campagnola, R.P. Russell, Endgame problem part 2: multibody technique and the Tisserand-Poincare graph, J. Guid. Control. Dyn. 33 (2010) 476-486.

[18] V. Maiwald, Applicability of Tisserand Criterion for Optimization of Gravity-assist Sequences for Low-thrust 
Missions, (2015).

[19] T.H. Cormen, C.E. Leiserson, R.L. Rivest, C. Stein, Introduction to algorithms, MIT press, 2009.

[20] D. Izzo, D. Hennes, L.F. Simões, M. Märtens, Designing complex interplanetary trajectories for the global trajectory optimization competitions, in: Sp. Eng., Springer, 2016: pp. 151-176.

[21] M. Vasile, P. De Pascale, Preliminary design of multiple gravity-assist trajectories, J. Spacecr. Rockets. 43 (2006) 794-805.

[22] R. Bellman, The theory of dynamic programming, Bull. Am. Math. Soc. 60 (1954) 503-515.

[23] F. Peralta, S. Flanagan, Cassini interplanetary trajectory design, Control Eng. Pract. 3 (1995) 1603-1610.

[24] B.A. Conway, Spacecraft trajectory optimization, Cambridge University Press, 2010.

[25] J. Englander, Automated trajectory planning for multiple-flyby interplanetary missions, (2013).

[26] T. Vinkó, D. Izzo, Global optimisation heuristics and test problems for preliminary spacecraft trajectory design, Adv. Concepts Team, ESATR ACT-TNT-MAD-GOHTPPSTD, Sept. (2008).

[27] J. Schoenmaekers, R. Bauske, Re-design of the Rosetta mission for launch in 2004, in: 18th Int. Symp. Sp. Flight Dyn., 2004: p. 227.

[28] B. Gladman, B.G. Marsden, C. VanLaerhoven, Nomenclature in the outer Solar System, Sol. Syst. Beyond Neptune. 43 (2008).

[29] O. Grasset, M.K. Dougherty, A. Coustenis, E.J. Bunce, C. Erd, D. Titov, M. Blanc, A. Coates, P. Drossart, L.N. Fletcher, JUpiter ICy moons Explorer (JUICE): An ESA mission to orbit Ganymede and to characterise the Jupiter system, Planet. Space Sci. 78 (2013) 1-21. 
$2021-12-29$

\title{
An automatic process for sample return missions based on dynamic programming optimization
}

\author{
Bellome, Andrea
}

AIAA

Bellome A, Sanchez Cuartielles J-P, Rico Alvarez JI, et al., (2021) An automatic process for sample return missions based on dynamic programming optimization. In: AIAA SciTech 2022

Forum, 3-7 January 2022, San Diego and Virtual Event, Paper number AIAA 2022-1477

https://doi.org/10.2514/6.2022-1477

Downloaded from Cranfield Library Services E-Repository 